\title{
Supporting Information Impedance Characterization and Modeling of Subcellular to Micro-Sized Electrodes with Varying Materials and PEDOT:PSS-Coating for Bioelectrical Interfaces
}

\author{
Adam Wang ${ }^{1, \hbar}$, Doohwan Jung ${ }^{1, *}$, Dongwon Lee ${ }^{1}$, Hua Wang ${ }^{1,2, *}$ \\ ${ }^{I}$ Department of Electrical and Computer Engineering, Georgia Institute of Technology, Atlanta, GA \\ 30332, USA \\ ${ }^{2}$ Deptartment of Information Technology and Electrical Engineering, ETH Zurich, Zurich 8092, \\ Switzerland \\ ${ }^{*}$ A.W. and D.J. contributed equally to this work.
}

*Correspondence: huawang@ethz.ch 


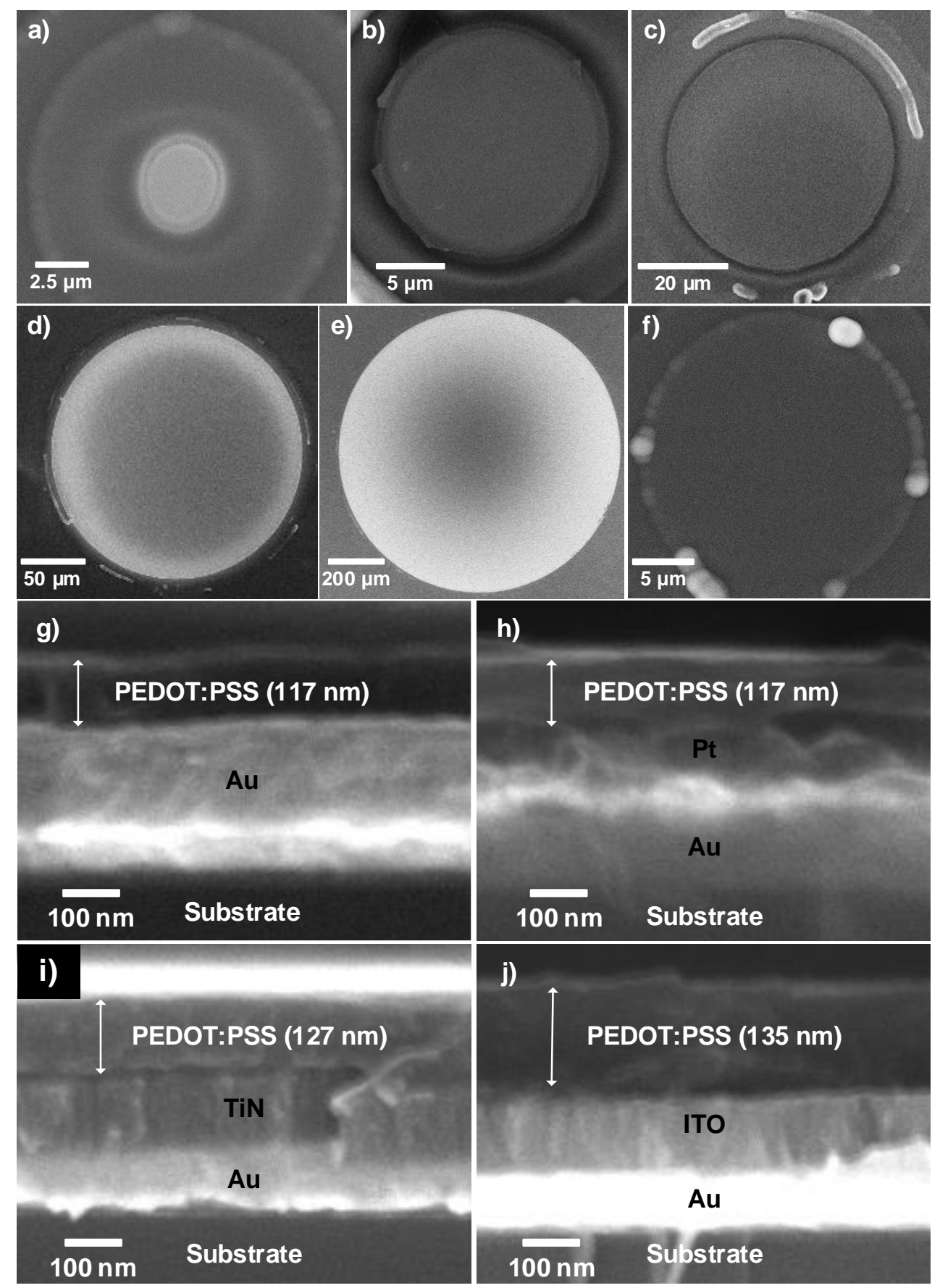

Figure S1. Additional morphology and structure of fabricated electrodes. Top view SEM images of PEDOT:PSS/Au microelectrodes with diameters of a) $2.5 \mu \mathrm{m}$, b) $15 \mu \mathrm{m}$, c) $20 \mu \mathrm{m}$, d) $200 \mu \mathrm{m}$, and e) $1000 \mu \mathrm{m}$. f) Top view SEM image of a parylene C passivated Au microelectrode with $\underline{D}=20 \mu \mathrm{m}$. Cross sectional views of PEDOT:PSS spin casted over g) Au, h) Pt, i) TiN, and j) ITO substrates. 


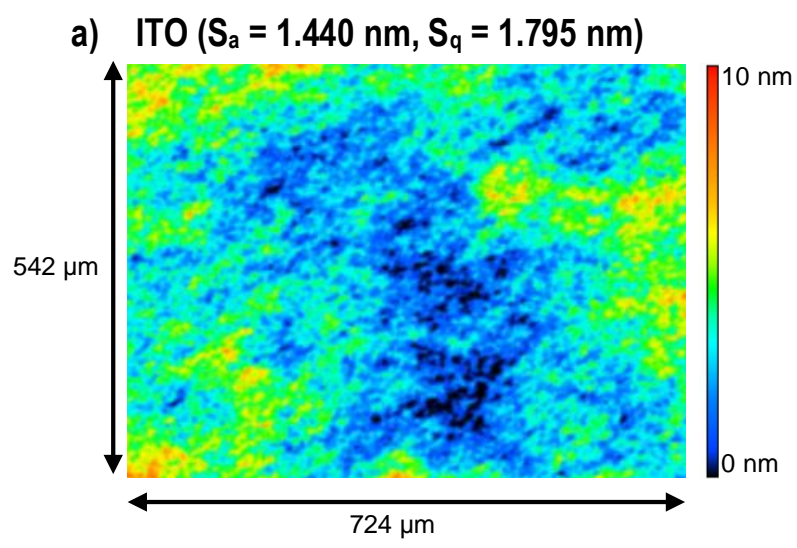

b) PEDOT:PSS/ITO $\left(\mathrm{S}_{\mathrm{a}}=1.879 \mathrm{~nm}, \mathrm{~S}_{\mathrm{q}}=2.398 \mathrm{~nm}\right)$

c) $\mathrm{Au}\left(\mathrm{S}_{\mathrm{a}}=1.473 \mathrm{~nm}, \mathrm{~S}_{\mathrm{q}}=1.844 \mathrm{~nm}\right)$
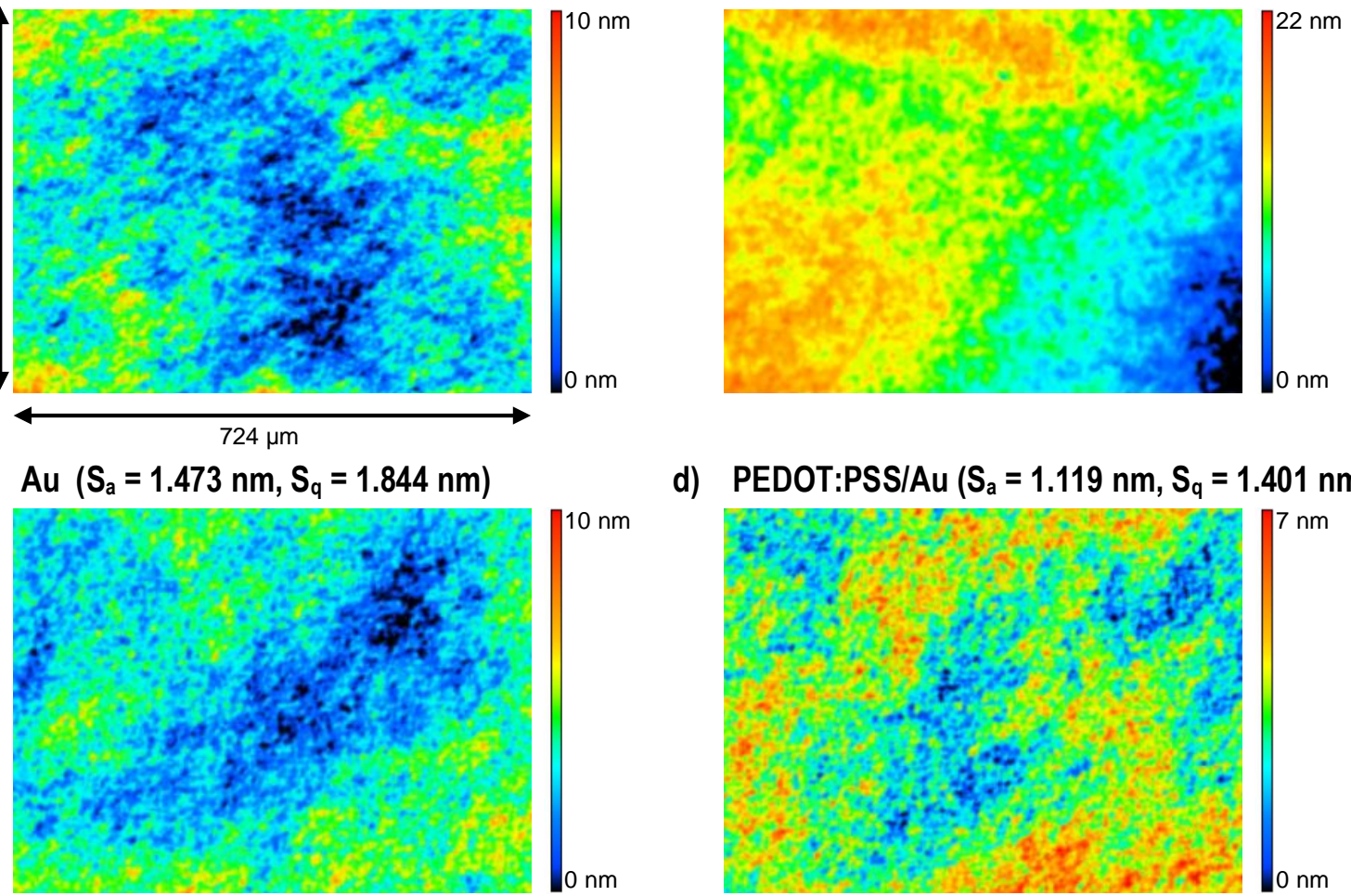

d) PEDOT:PSS $/ A u\left(S_{a}=1.119 \mathrm{~nm}, S_{q}=1.401 \mathrm{~nm}\right)$

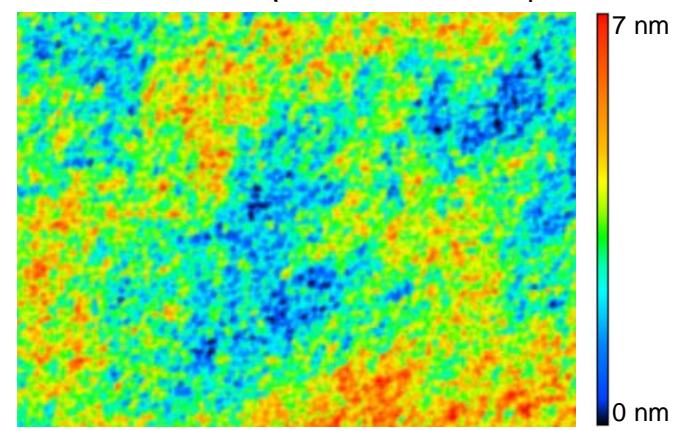

e) $\operatorname{Pt}\left(S_{a}=1.054 \mathrm{~nm}, S_{q}=1.321 \mathrm{~nm}\right)$

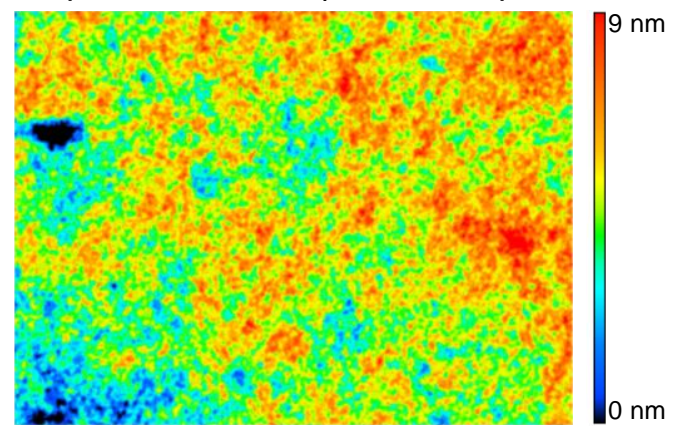

f) PEDOT:PSS/Pt $\left(S_{a}=1.215 \mathrm{~nm}, S_{q}=1.666 \mathrm{~nm}\right)$

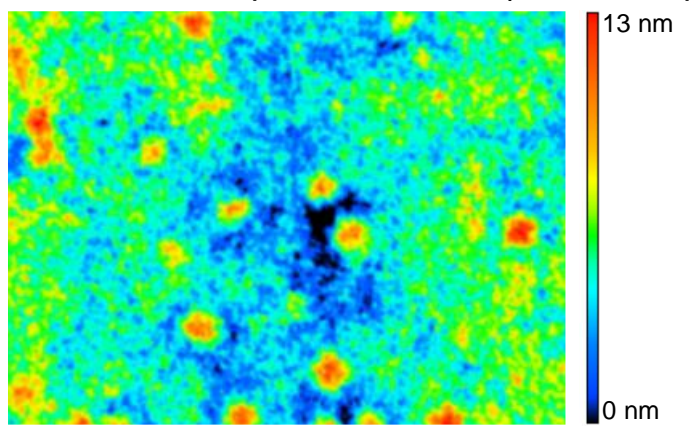

g) $\operatorname{TiN}\left(S_{a}=1.665 \mathrm{~nm}, S_{q}=2.094 \mathrm{~nm}\right)$

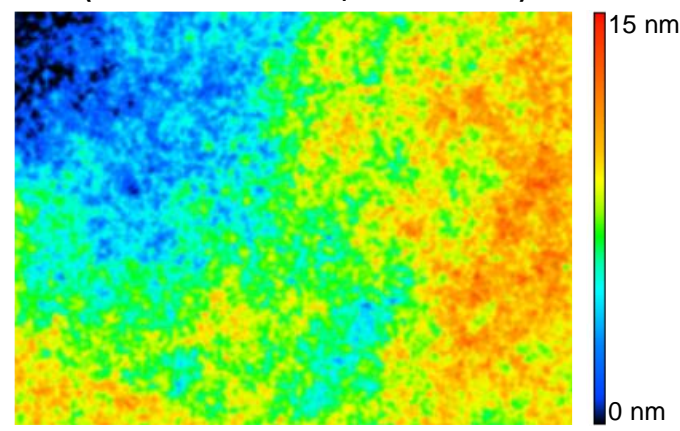

h) PEDOT:PSS/TiN $\left(S_{a}=1.477 \mathrm{~nm}, S_{q}=1.845 \mathrm{~nm}\right)$

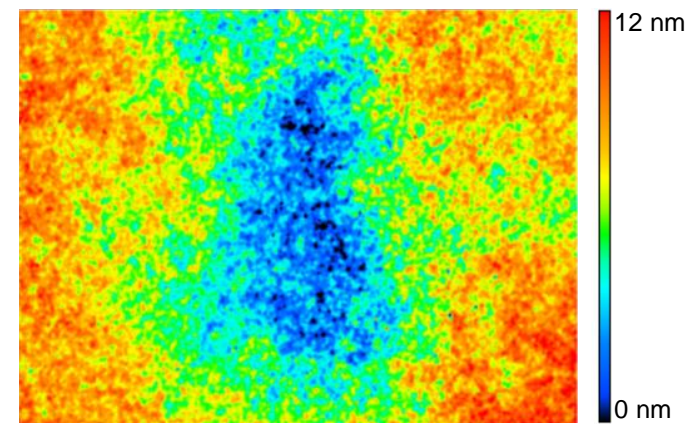

Figure S2. Surface roughness images taken with light interferometry. Arithmetical mean and root mean square height for bare and PEDOT:PSS coated a,b) ITO, c,d) $\mathrm{Au}, \mathbf{e , f}) \mathrm{Pt}$, and $\mathbf{g , h}$ ) TiN, respectively. 

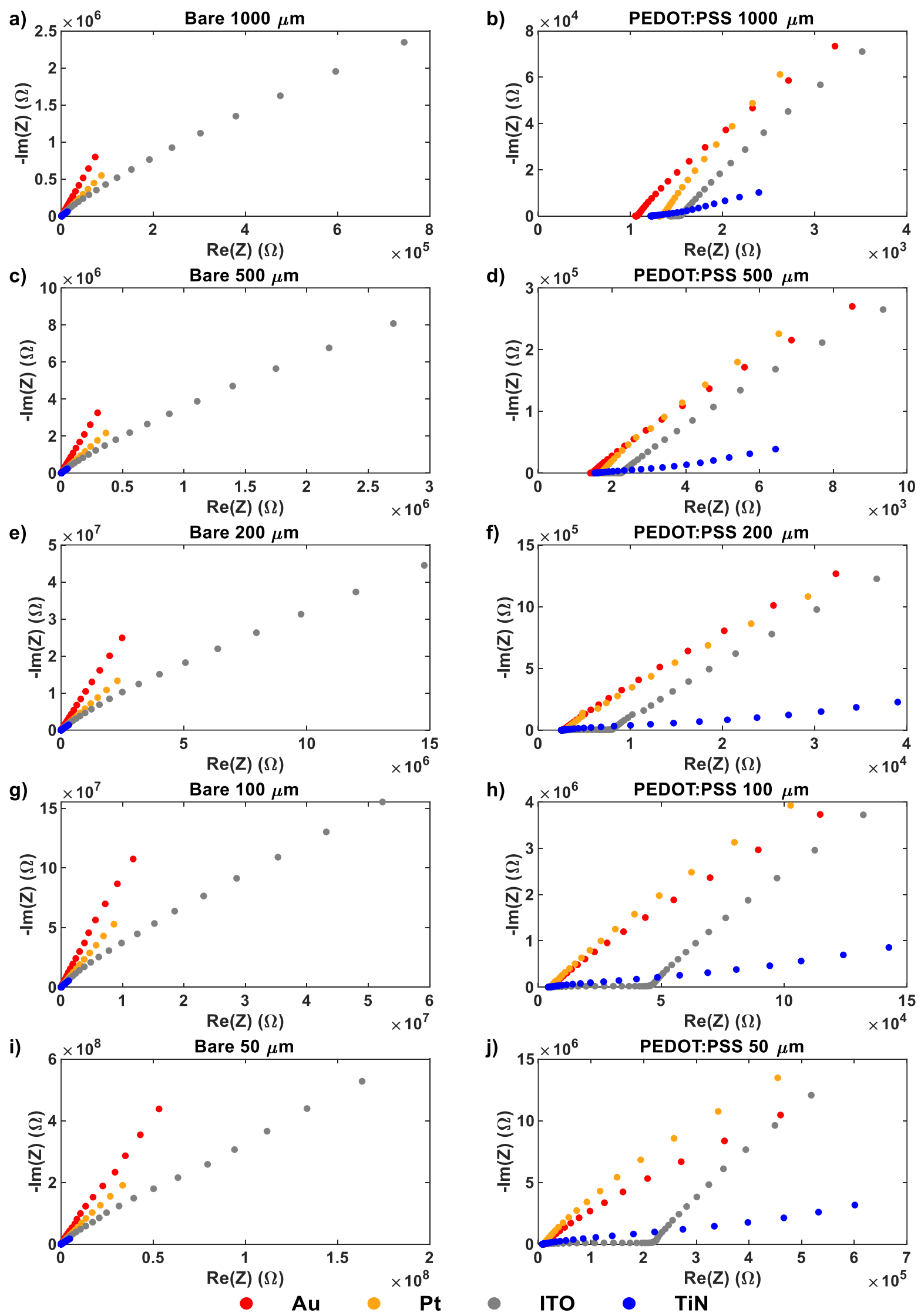

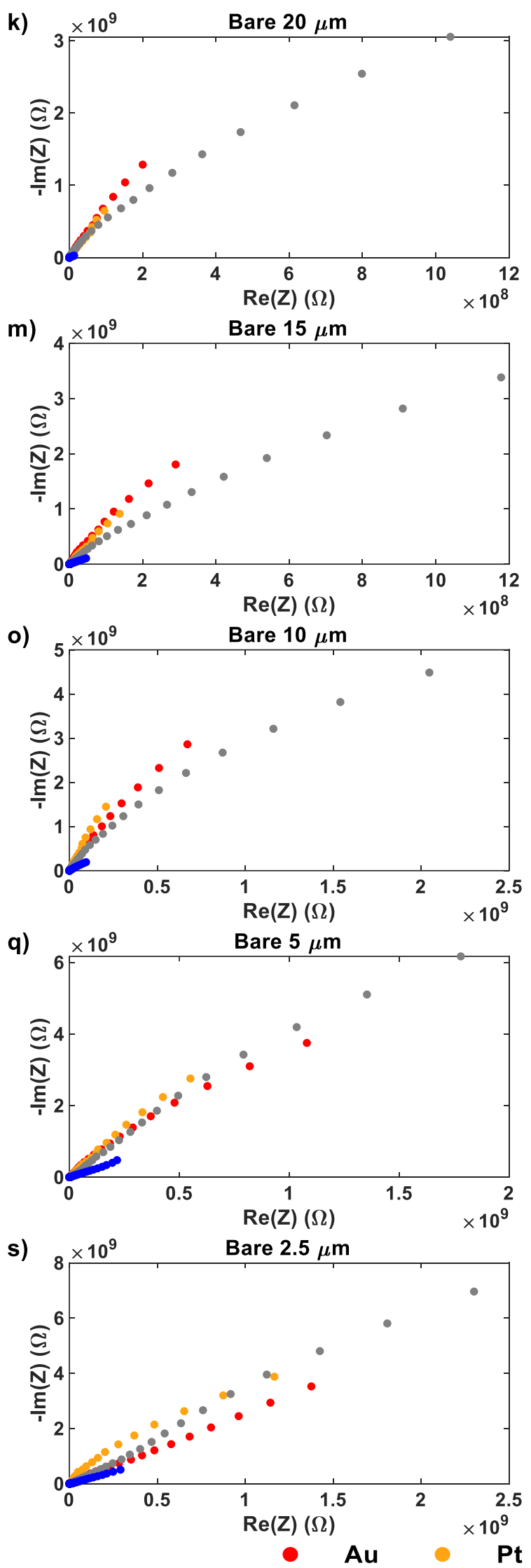
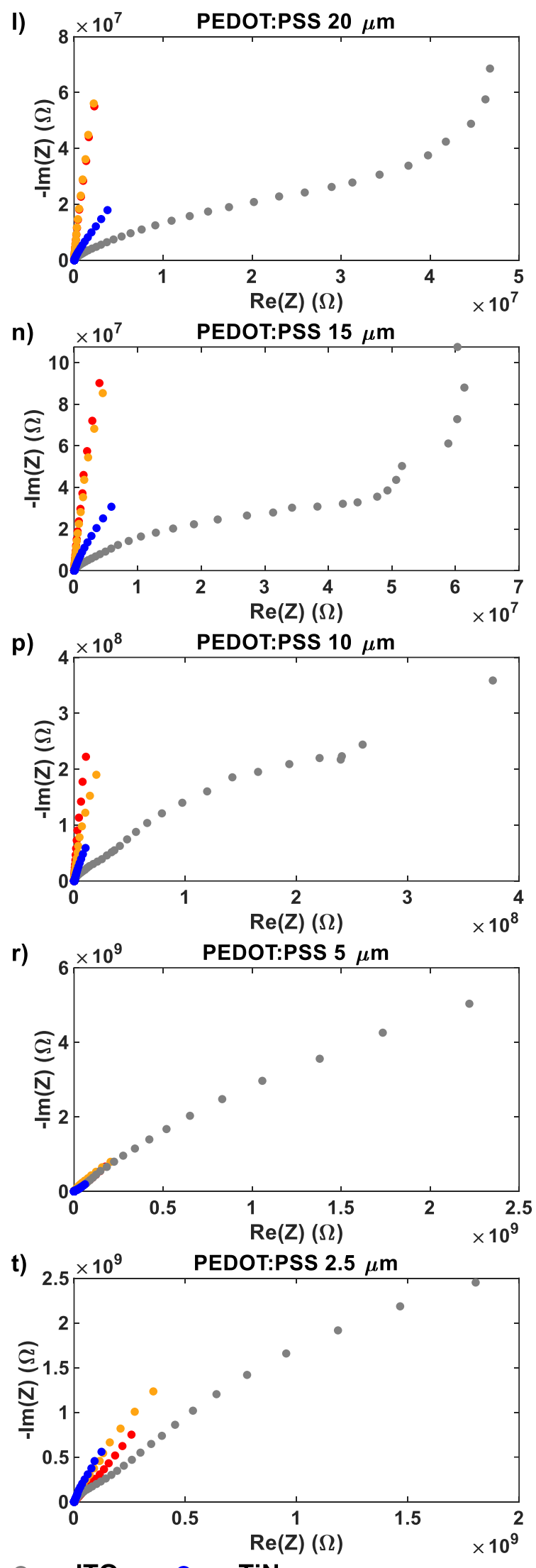
Figure S3. Nyquist plots of bare and PEDOT:PSS coated Au, Pt, ITO, and TiN electrodes with different sizes ranging from $D=2.5$ to $1000 \mu \mathrm{m}$. Bare and PEDOT:PSS electrodes, respectively with $D=\mathbf{a , b ) ~} 1000 \mu \mathrm{m}, \mathbf{c}, \mathbf{d}) 500 \mu \mathrm{m}, \mathbf{e , f}) 200 \mu \mathrm{m}, \mathbf{g}, \mathbf{h}) 100 \mu \mathrm{m}, \mathbf{i}, \mathbf{j}) 50 \mu \mathrm{m}, \mathbf{k}, \mathbf{l}) 20$ $\mu \mathrm{m}, \mathbf{m}, \mathbf{n}) 15 \mu \mathrm{m}, \mathbf{o , p )} 10 \mu \mathrm{m}, \mathbf{q}, \mathbf{r}) 5 \mu \mathrm{m}$, and $\mathbf{s , t}) 2.5 \mu \mathrm{m}$. 
a)

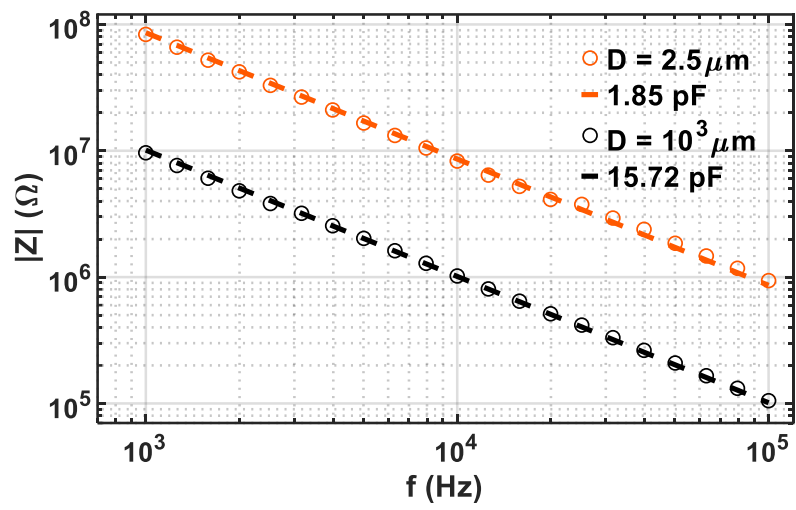

c)

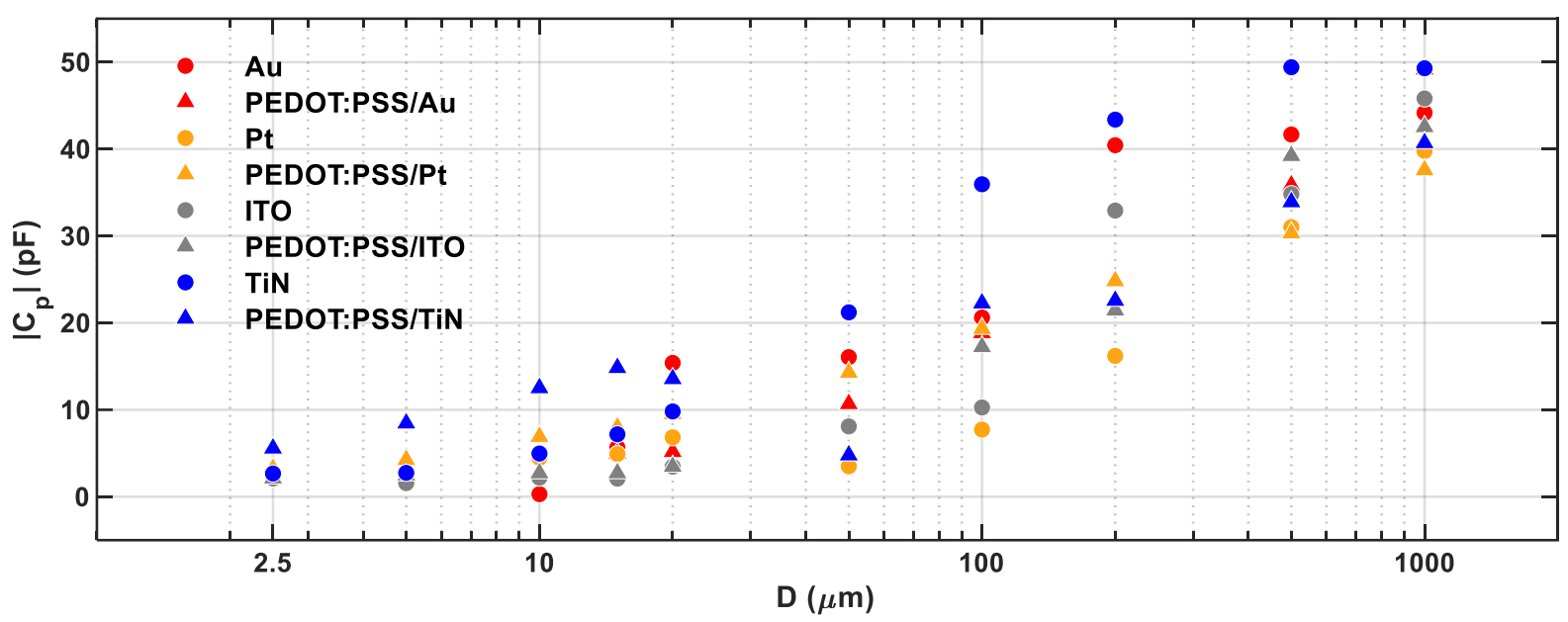

b)

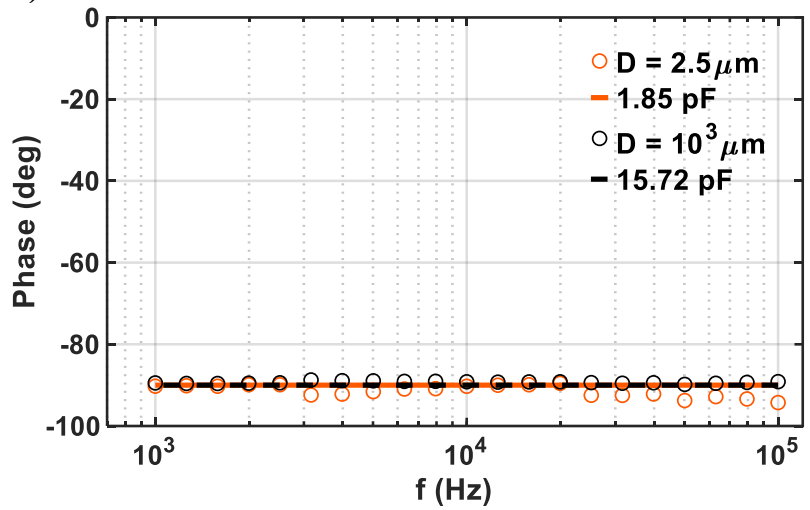

Figure S4. Parasitic capacitance analysis of passivated and opened electrodes. EIS plots of $D=2.5$ and $1000 \mu \mathrm{m}$ passivated electrodes where measured data (open circle) is fitted (dotted line) using a capacitor over a) Impedance magnitude and b) phase plots which shows the parasitic capacitance of passivated electrodes ranges from $1.85 \mathrm{pF}$ to $15.72 \mathrm{pF}$. c) The extracted parasitic capacitances of opened electrodes exhibit a positive correlation with size. 
(a)

(b)
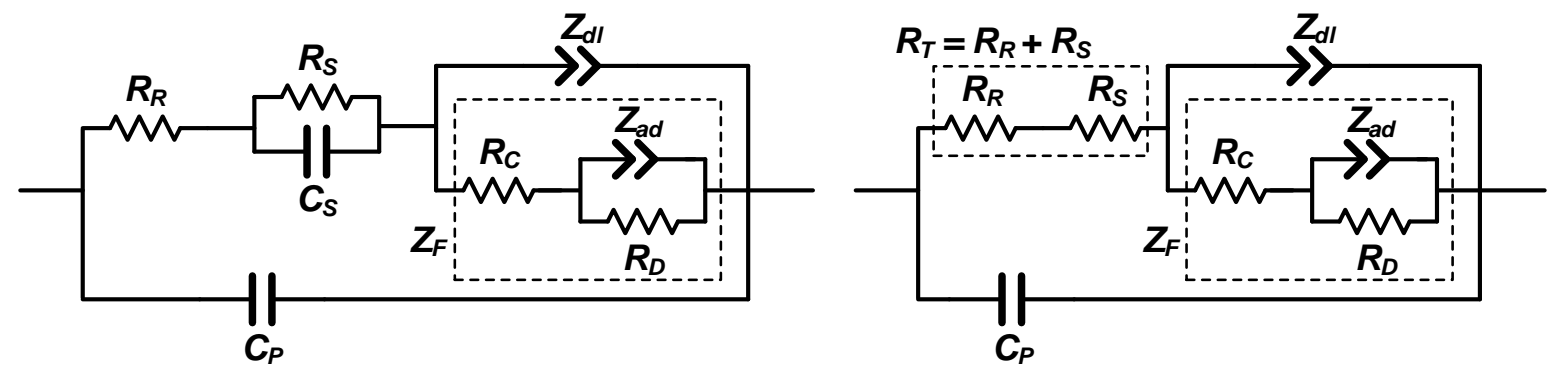

Figure S5. a) Equivalent circuit model with a solution capacitance $\mathrm{C}_{\mathrm{S}}$. b) Proposed equivalent circuit model with neglecting the solution capacitance $\mathrm{C}_{\mathrm{S}}$.

The proposed equivalent circuit model in Figure S5b has a transfer function $T(s)$ where $s=j \omega$ such that

$T(s)=\frac{N(s)}{D(s)}$

$N(s)=R_{C}+R_{D}+R_{T}+Q_{a d} R_{C} R_{D} s^{n_{a d}}+Q_{a d} R_{D} R_{T} s^{n_{a d}}+Q_{d l} R_{C} R_{T} s^{n_{d l}}+Q_{d l} R_{D} R_{T} s^{n_{d l}}+$ $Q_{a d} Q_{d l} R_{C} R_{D} R_{T} S^{n_{a d}+n_{d l}}$

$D(s)=1+s\left(C_{P} R_{C}+C_{P} R_{D}+C_{P} R_{T}\right)+Q_{a d} R_{D} s^{n_{a d}}+s^{1+n_{a d}}\left(C_{P} Q_{a d} R_{C} R_{D}+C_{P} Q_{a d} R_{D} R_{T}\right)+$ $s^{n d l}\left(Q_{d l} R_{C}+Q_{d l} R_{D}\right)+s^{1+n_{d l}}\left(C_{P} Q_{d l} R_{C} R_{T}+C_{P} Q_{d l} R_{D} R_{T}\right)+Q_{a d} Q_{d l} R_{C} R_{D} s^{n_{a d}+n_{d l}}+$ $C_{P} Q_{a d} Q_{d l} R_{C} R_{D} R_{T} S^{1+n_{a d}+n_{d l}}$

Due to the complex nature of the CPE, we will assume that $n=1$ for $n_{a d}$ and $n_{d l}$ to simplify analysis of the circuit response in which we will see that

$N(s)=R_{C}+R_{D}+R_{T}+s\left(Q_{a d} R_{C} R_{D}+Q_{a d} R_{D} R_{T}+Q_{d l} R_{C} R_{T}+Q_{d l} R_{D} R_{T}\right)+s^{2} Q_{a d} Q_{d l} R_{C} R_{D} R_{T}$

$D(s)=1+s\left(C_{P} R_{C}+Q_{d l} R_{C}+C_{P} R_{D}+Q_{a d} R_{D}+Q_{d l} R_{D}+C_{P} R_{T}\right)+s^{2}\left(C_{P} Q_{a d} R_{C} R_{D}+Q_{a d} Q_{d l} R_{C} R_{D}+\right.$ $\left.C_{P} Q_{d l} R_{C} R_{T}+C_{P} Q_{a d} R_{D} R_{T}+C_{P} Q_{d l} R_{D} R_{T}\right)+s^{3} C_{P} Q_{a d} Q_{d l} R_{C} R_{D} R_{T}$

Here, we can clearly see that this transfer function has 3 poles and 2 zeros with the starting phase at $0^{\circ}$ at very low frequencies $(<<1) \mathrm{mHz}$ with an initial magnitude of $R_{C}+R_{D}+R_{T}$. The first pole (at low frequencies) is caused by the faradaic adsorption capacitance $Z_{a d}$ which is then followed by a zero due to $R_{C}$. Afterwards, at higher frequencies another pole-zero pair in introducted with a pole initiated from the nonfaradaic double layer capacitance $Z_{d l}$ followed by a zero originating from the solution and routing resistance $R_{T}$. At much higher frequencies $(>70$ $\mathrm{kHz}$ ) we can observe the effects of the last pole derived from the parasitic capacitance $C_{P}$. The presence of these pole-zero pairs are very apparent in the example of $100 \mu \mathrm{m}$ PEDOT:PSS/ITO electrode where we can see the phase increase (zero) and decrease (pole) when transitioning at around $500 \mathrm{~Hz}$ and then an increase (zero) in phase near $10 \mathrm{kHz}$. If the peaking and dipping of the phase is not easily observed at two areas it is due to pole-zero cancellations where a pole and zero occur very closely in the frequency spectrum. 

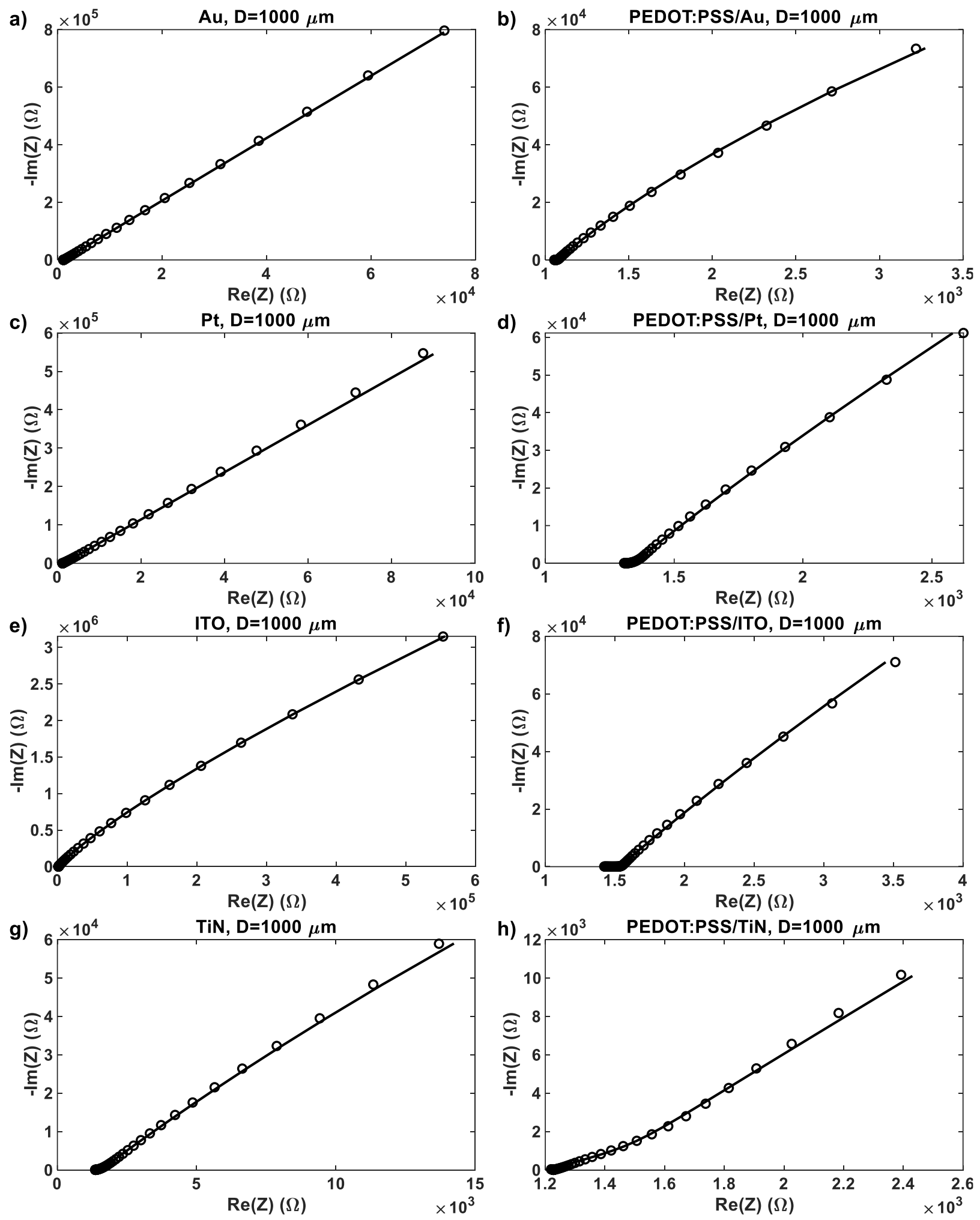

Figure S6. Fitting Nyquist plots of bare and PEDOT:PSS coated, respectively, a,b) $\mathrm{Au}, \mathbf{c}, \mathbf{d}) \mathrm{Pt}$, e,f) ITO, and g,h) TiN electrodes with $D=1000 \mu \mathrm{m}$ using equivalent circuit models. 

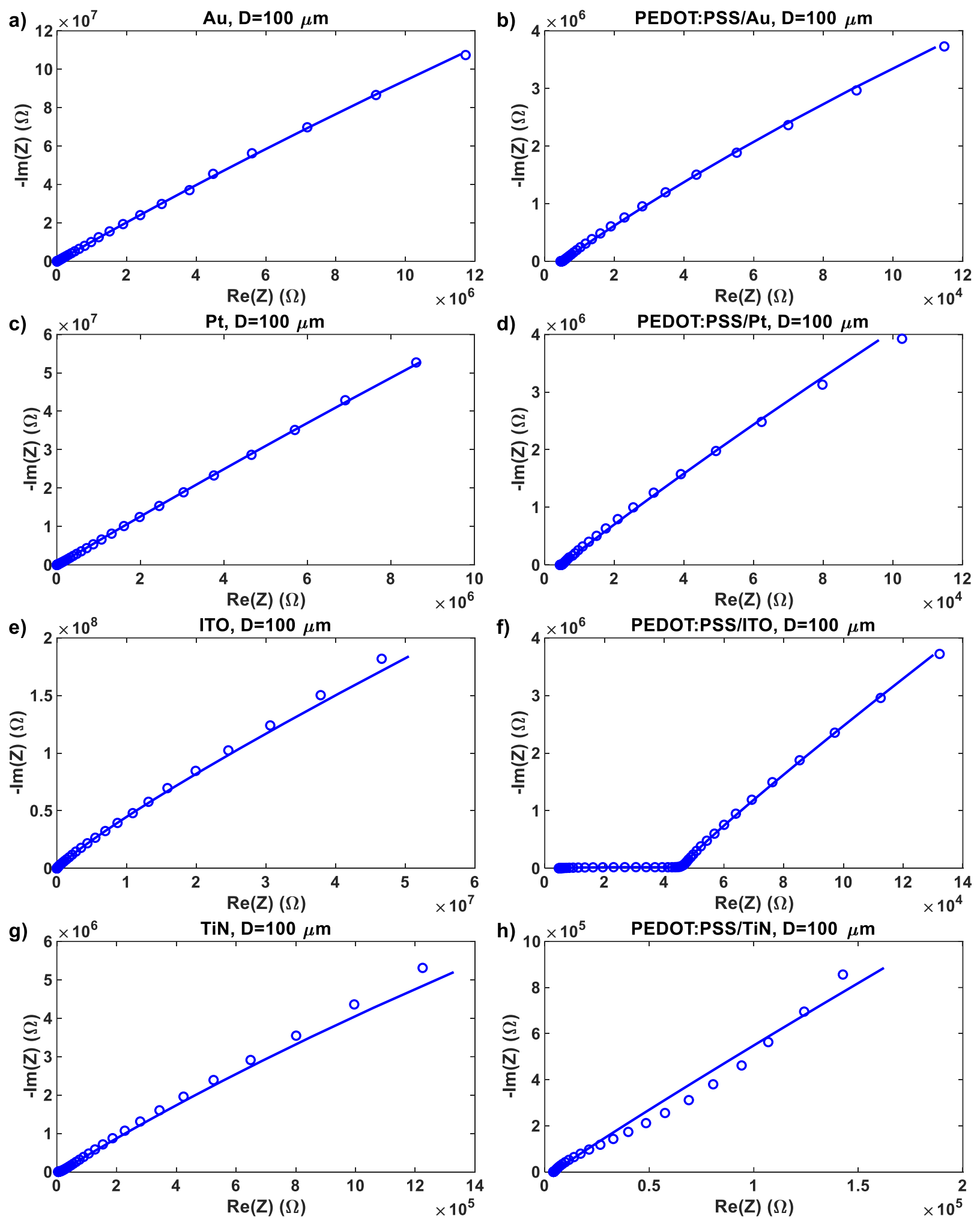

Figure S7. Fitting Nyquist plots of bare and PEDOT:PSS coated, respectively, a,b) Au, c,d) Pt, e,f) ITO, and g,h) TiN electrodes with $D=100 \mu \mathrm{m}$ using equivalent circuit models. 

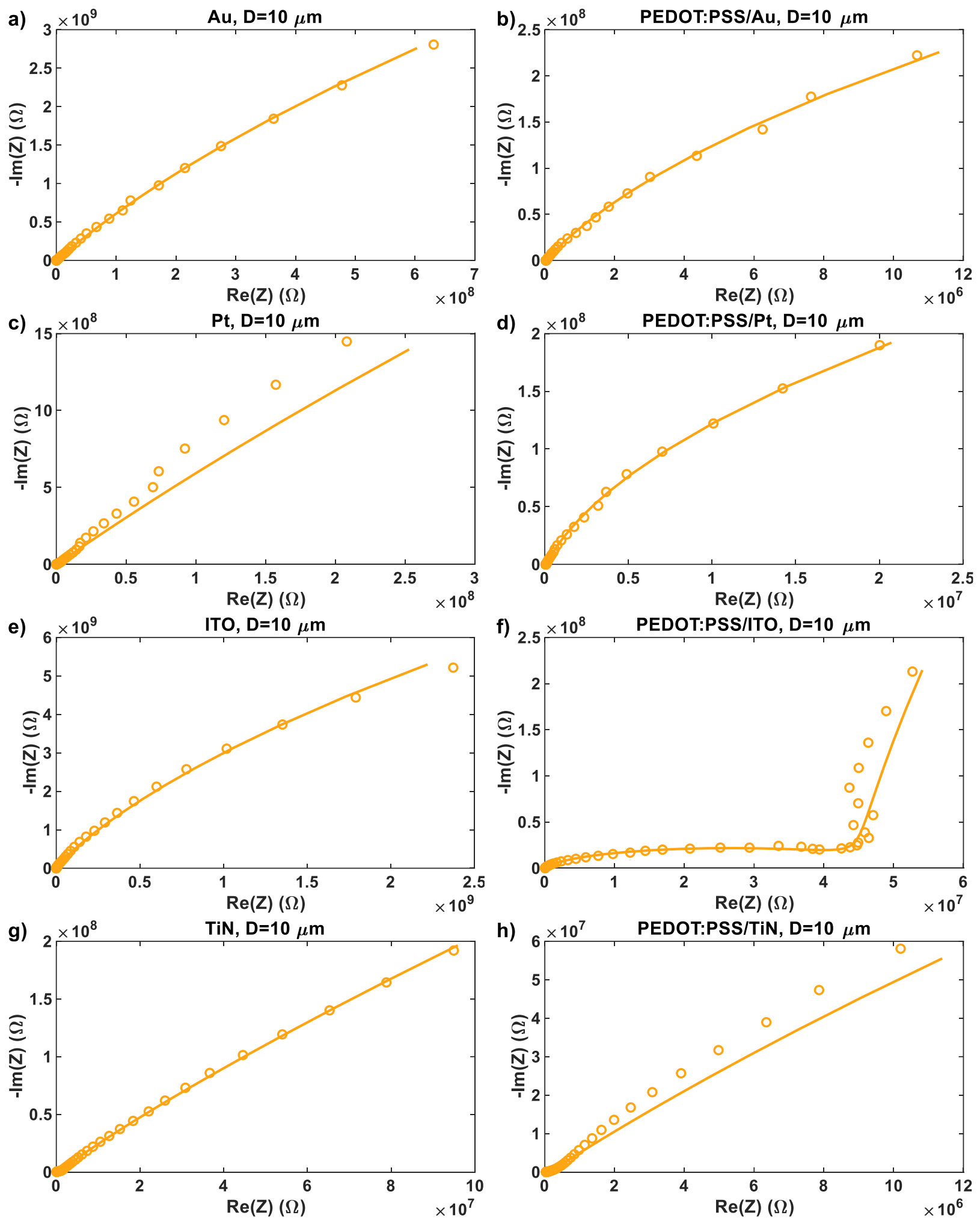

Figure S8. Fitting Nyquist plots of bare and PEDOT:PSS coated, respectively, a,b) $\mathrm{Au}, \mathbf{c}, \mathbf{d}) \mathrm{Pt}$, e,f) ITO, and $\mathbf{g , h}$ ) TiN electrodes with $D=10 \mu \mathrm{m}$ using equivalent circuit models. 
Table S1. Extracted equivalent circuit component values for bare Au electrodes.

\begin{tabular}{ccccccccc}
\hline $\begin{array}{c}D \\
{[\mu \mathrm{m}]}\end{array}$ & $\begin{array}{c}R_{S}+R_{R} \\
{[\Omega]}\end{array}$ & $\begin{array}{c}Q_{d l} \\
{\left[\mathrm{~F} \cdot \mathrm{s}^{\mathrm{n}-1}\right]}\end{array}$ & $n_{d l}$ & $\begin{array}{c}R_{C} \\
{[\Omega]}\end{array}$ & $\begin{array}{c}Q_{a d} \\
{\left[\mathrm{~F} \cdot \mathrm{s}^{\mathrm{n}-1}\right]}\end{array}$ & $n_{a d}$ & $\begin{array}{c}R_{D} \\
{[\Omega]}\end{array}$ & $\begin{array}{c}C_{P} \\
{[\mathrm{~F}]}\end{array}$ \\
\hline 2.5 & $1.23 \mathrm{e} 5$ & $6.08 \mathrm{e}-11$ & 0.73 & $3.98 \mathrm{e} 7$ & $2.05 \mathrm{e}-12$ & 0.85 & $4.82 \mathrm{e} 10$ & $2.06 \mathrm{e}-12$ \\
5 & $6.30 \mathrm{e} 4$ & $4.42 \mathrm{e}-11$ & 0.88 & $3.68 \mathrm{e} 6$ & $2.53 \mathrm{e}-11$ & 0.85 & $4.50 \mathrm{e} 10$ & $2.37 \mathrm{e}-12$ \\
10 & $3.20 \mathrm{e} 4$ & $5.03 \mathrm{e}-11$ & 0.91 & $2.51 \mathrm{e} 6$ & $1.56 \mathrm{e}-11$ & 0.88 & $4.22 \mathrm{e} 10$ & $2.93 \mathrm{e}-13$ \\
15 & $2.30 \mathrm{e} 4$ & $8.12 \mathrm{e}-11$ & 0.92 & $5.42 \mathrm{e} 7$ & $2.38 \mathrm{e}-12$ & 1 & $6.56 \mathrm{e} 10$ & $5.66 \mathrm{e}-12$ \\
20 & $1.78 \mathrm{e} 4$ & $1.06 \mathrm{e}-10$ & 0.90 & $4.08 \mathrm{e} 6$ & $2.24 \mathrm{e}-12$ & 1 & $4.10 \mathrm{e} 10$ & $1.54 \mathrm{e}-11$ \\
50 & $6.24 \mathrm{e} 3$ & $3.20 \mathrm{e}-10$ & 0.94 & $3.08 \mathrm{e} 6$ & $7.39 \mathrm{e}-11$ & 0.81 & $3.11 \mathrm{e} 10$ & $1.61 \mathrm{e}-11$ \\
100 & $3.08 \mathrm{e} 3$ & $1.55 \mathrm{e}-9$ & 0.93 & $1.69 \mathrm{e} 6$ & $6.92 \mathrm{e}-11$ & 0.94 & $1.01 \mathrm{e} 10$ & $2.06 \mathrm{e}-11$ \\
200 & $2.02 \mathrm{e} 3$ & $6.41 \mathrm{e}-9$ & 0.94 & $1.29 \mathrm{e} 5$ & $7.65 \mathrm{e}-10$ & 0.92 & $7.22 \mathrm{e} 9$ & $4.04 \mathrm{e}-11$ \\
500 & $1.35 \mathrm{e} 3$ & $4.54 \mathrm{e}-8$ & 0.95 & $4.55 \mathrm{e} 3$ & $9.20 \mathrm{e}-9$ & 0.92 & $8.75 \mathrm{e} 8$ & $4.17 \mathrm{e}-11$ \\
1000 & $1.14 \mathrm{e} 3$ & $1.84 \mathrm{e}-7$ & 0.95 & $2.12 \mathrm{e} 3$ & $3.95 \mathrm{e}-8$ & 0.92 & $1.48 \mathrm{e} 9$ & $4.42 \mathrm{e}-11$ \\
\hline
\end{tabular}

Table S2. Extracted equivalent circuit component values for PEDOT:PSS/Au electrodes.

\begin{tabular}{ccccccccc}
$\begin{array}{c}D \\
{[\mu \mathrm{m}]}\end{array}$ & $\begin{array}{c}R_{S}+R_{R} \\
{[\Omega]}\end{array}$ & $\begin{array}{c}Q_{d l} \\
{\left[\mathrm{~F} \cdot \mathrm{s}^{\mathrm{n}-1}\right]}\end{array}$ & $n_{d l}$ & $\begin{array}{c}R_{C} \\
{[\Omega]}\end{array}$ & $\begin{array}{c}Q_{a d} \\
{\left[\mathrm{~F} \cdot \mathrm{s}^{\mathrm{n}-1}\right]}\end{array}$ & $n_{a d}$ & $\begin{array}{c}R_{D} \\
{[\Omega]}\end{array}$ & $\begin{array}{c}C_{P} \\
{[\mathrm{~F}]}\end{array}$ \\
\hline 2.5 & $1.26 \mathrm{e} 5$ & $1.52 \mathrm{e}-10$ & 0.72 & $1.12 \mathrm{e} 6$ & $1.21 \mathrm{e}-10$ & 0.99 & $1.55 \mathrm{e} 10$ & $2.24 \mathrm{e}-12$ \\
5 & $9.41 \mathrm{e} 4$ & $2.59 \mathrm{e}-10$ & 0.82 & $1.02 \mathrm{e} 6$ & $3.84 \mathrm{e}-11$ & 0.99 & $1.08 \mathrm{e} 10$ & $3.22 \mathrm{e}-12$ \\
10 & $3.24 \mathrm{e} 4$ & $3.42 \mathrm{e}-10$ & 1 & $2.37 \mathrm{e} 4$ & $3.77 \mathrm{e}-10$ & 0.97 & $8.88 \mathrm{e} 9$ & $4.81 \mathrm{e}-12$ \\
15 & $2.25 \mathrm{e} 4$ & $9.24 \mathrm{e}-10$ & 1 & $1.30 \mathrm{e} 4$ & $8.60 \mathrm{e}-10$ & 0.97 & $4.17 \mathrm{e} 9$ & $4.98 \mathrm{e}-12$ \\
20 & $1.76 \mathrm{e} 4$ & $1.58 \mathrm{e}-9$ & 1 & $9.22 \mathrm{e} 3$ & $1.34 \mathrm{e}-9$ & 0.97 & $2.37 \mathrm{e} 9$ & $5.16 \mathrm{e}-12$ \\
50 & $1.01 \mathrm{e} 4$ & $6.10 \mathrm{e}-9$ & 1 & $4.04 \mathrm{e} 3$ & $9.70 \mathrm{e}-9$ & 0.97 & $6.69 \mathrm{e} 8$ & $1.07 \mathrm{e}-11$ \\
100 & $4.61 \mathrm{e} 3$ & $2.12 \mathrm{e}-8$ & 1 & $1.89 \mathrm{e} 3$ & $2.28 \mathrm{e}-8$ & 0.97 & $7.61 \mathrm{e} 8$ & $1.88 \mathrm{e}-11$ \\
200 & $2.56 \mathrm{e} 3$ & $7.57 \mathrm{e}-8$ & 1 & $9.51 \mathrm{e} 2$ & $5.27 \mathrm{e}-8$ & 0.97 & $3.29 \mathrm{e} 8$ & $2.15 \mathrm{e}-11$ \\
500 & $1.42 \mathrm{e} 3$ & $4.02 \mathrm{e}-7$ & 1 & $4.43 \mathrm{e} 2$ & $2.01 \mathrm{e}-7$ & 0.96 & $4.44 \mathrm{e} 7$ & $3.58 \mathrm{e}-11$ \\
1000 & $1.06 \mathrm{e} 3$ & $1.43 \mathrm{e}-6$ & 1 & $1.47 \mathrm{e} 2$ & $7.91 \mathrm{e}-7$ & 0.96 & $8.42 \mathrm{e} 6$ & $4.92 \mathrm{e}-11$ \\
\hline
\end{tabular}


Table S3. Extracted equivalent circuit component values for bare Pt electrodes.

\begin{tabular}{ccccccccc}
\hline $\begin{array}{c}D \\
{[\mu \mathrm{m}]}\end{array}$ & $\begin{array}{c}R_{S}+R_{R} \\
{[\Omega]}\end{array}$ & $\begin{array}{c}Q_{d l} \\
{\left[\mathrm{~F} \cdot \mathrm{s}^{\mathrm{n}-1}\right]}\end{array}$ & $n_{d l}$ & $\begin{array}{c}R_{C} \\
{[\Omega]}\end{array}$ & $\begin{array}{c}Q_{a d} \\
{\left[\mathrm{~F} \cdot \mathrm{s}^{\mathrm{n}-1}\right]}\end{array}$ & $n_{a d}$ & $\begin{array}{c}R_{D} \\
{[\Omega]}\end{array}$ & $\begin{array}{c}C_{P} \\
{[\mathrm{~F}]}\end{array}$ \\
\hline 2.5 & $1.03 \mathrm{e} 5$ & $2.54 \mathrm{e}-11$ & 0.88 & $6.29 \mathrm{e} 6$ & $1.93 \mathrm{e}-11$ & 0.86 & $3.94 \mathrm{e} 10$ & $2.25 \mathrm{e}-12$ \\
5 & $5.20 \mathrm{e} 4$ & $5.57 \mathrm{e}-11$ & 0.87 & $1.78 \mathrm{e} 7$ & $1.54 \mathrm{e}-11$ & 0.86 & $1.86 \mathrm{e} 11$ & $2.80 \mathrm{e}-12$ \\
10 & $2.68 \mathrm{e} 4$ & $9.78 \mathrm{e}-11$ & 0.89 & $7.82 \mathrm{e} 6$ & $3.24 \mathrm{e}-11$ & 0.91 & $7.04 \mathrm{e} 10$ & $4.48 \mathrm{e}-12$ \\
15 & $1.63 \mathrm{e} 4$ & $1.63 \mathrm{e}-10$ & 0.89 & $7.03 \mathrm{e} 6$ & $4.12 \mathrm{e}-11$ & 0.92 & $5.05 \mathrm{e} 10$ & $4.90 \mathrm{e}-12$ \\
20 & $1.21 \mathrm{e} 4$ & $2.31 \mathrm{e}-10$ & 0.90 & $7.71 \mathrm{e} 6$ & $5.46 \mathrm{e}-11$ & 0.90 & $3.06 \mathrm{e} 10$ & $6.83 \mathrm{e}-12$ \\
50 & $4.93 \mathrm{e} 3$ & $7.03 \mathrm{e}-10$ & 0.93 & $3.24 \mathrm{e} 6$ & $3.06 \mathrm{e}-10$ & 0.79 & $2.15 \mathrm{e} 11$ & $3.50 \mathrm{e}-12$ \\
100 & $2.94 \mathrm{e} 3$ & $2.95 \mathrm{e}-9$ & 0.91 & $2.07 \mathrm{e} 6$ & $6.21 \mathrm{e}-10$ & 0.82 & $7.82 \mathrm{e} 9$ & $7.72 \mathrm{e}-12$ \\
200 & $1.99 \mathrm{e} 3$ & $1.23 \mathrm{e}-8$ & 0.91 & $6.70 \mathrm{e} 5$ & $2.14 \mathrm{e}-9$ & 0.84 & $1.65 \mathrm{e} 9$ & $1.62 \mathrm{e}-11$ \\
500 & $1.41 \mathrm{e} 3$ & $8.16 \mathrm{e}-8$ & 0.89 & $1.84 \mathrm{e} 5$ & $6.78 \mathrm{e}-9$ & 0.88 & $3.48 \mathrm{e} 9$ & $3.10 \mathrm{e}-11$ \\
1000 & $1.22 \mathrm{e} 3$ & $3.17 \mathrm{e}-7$ & 0.90 & $3.64 \mathrm{e} 4$ & $3.12 \mathrm{e}-8$ & 0.89 & $2.08 \mathrm{e} 9$ & $3.98 \mathrm{e}-11$ \\
\hline
\end{tabular}

Table S4. Extracted equivalent circuit component values for PEDOT:PSS/Pt electrodes.

\begin{tabular}{ccccccccc}
$\begin{array}{c}D \\
{[\mu \mathrm{m}]}\end{array}$ & $\begin{array}{c}R_{S}+R_{R} \\
{[\Omega]}\end{array}$ & $\begin{array}{c}Q_{d l} \\
{\left[\mathrm{~F} \cdot \mathrm{s}^{\mathrm{n}-1}\right]}\end{array}$ & $n_{d l}$ & $\begin{array}{c}R_{C} \\
{[\Omega]}\end{array}$ & $\begin{array}{c}Q_{a d} \\
{\left[\mathrm{~F} \cdot \mathrm{s}^{\mathrm{n}-1}\right]}\end{array}$ & $n_{a d}$ & $\begin{array}{c}R_{D} \\
{[\Omega]}\end{array}$ & $\begin{array}{c}C_{P} \\
{[\mathrm{~F}]}\end{array}$ \\
\hline 2.5 & $2.08 \mathrm{e} 5$ & $6.16 \mathrm{e}-11$ & 0.82 & $2.32 \mathrm{e} 6$ & $9.79 \mathrm{e}-11$ & 0.89 & $2.75 \mathrm{e} 10$ & $3.21 \mathrm{e}-12$ \\
5 & $1.12 \mathrm{e} 5$ & $9.75 \mathrm{e}-11$ & 0.86 & $1.15 \mathrm{e} 6$ & $1.44 \mathrm{e}-10$ & 0.89 & $1.53 \mathrm{e} 10$ & $4.24 \mathrm{e}-12$ \\
10 & $5.11 \mathrm{e} 4$ & $1.14 \mathrm{e}-10$ & 1 & $2.87 \mathrm{e} 4$ & $7.35 \mathrm{e}-10$ & 0.97 & $2.76 \mathrm{e} 9$ & $6.90 \mathrm{e}-12$ \\
15 & $2.72 \mathrm{e} 4$ & $4.47 \mathrm{e}-10$ & 1 & $1.12 \mathrm{e} 4$ & $1.43 \mathrm{e}-9$ & 0.98 & $2.84 \mathrm{e} 9$ & $7.97 \mathrm{e}-12$ \\
20 & $1.93 \mathrm{e} 4$ & $1.07 \mathrm{e}-9$ & 1 & $8.07 \mathrm{e} 3$ & $1.79 \mathrm{e}-9$ & 0.98 & $2.59 \mathrm{e} 9$ & $9.53 \mathrm{e}-12$ \\
50 & $8.51 \mathrm{e} 3$ & $6.35 \mathrm{e}-9$ & 1 & $6.91 \mathrm{e} 3$ & $5.88 \mathrm{e}-9$ & 0.97 & $1.40 \mathrm{e} 9$ & $1.43 \mathrm{e}-11$ \\
100 & $4.45 \mathrm{e} 3$ & $2.03 \mathrm{e}-8$ & 1 & $1.72 \mathrm{e} 3$ & $2.15 \mathrm{e}-8$ & 0.97 & $2.51 \mathrm{e} 9$ & $1.93 \mathrm{e}-11$ \\
200 & $2.75 \mathrm{e} 3$ & $7.61 \mathrm{e}-8$ & 1 & $7.11 \mathrm{e} 2$ & $7.45 \mathrm{e}-8$ & 0.97 & $2.92 \mathrm{e} 8$ & $2.48 \mathrm{e}-11$ \\
500 & $1.66 \mathrm{e} 3$ & $4.57 \mathrm{e}-7$ & 1 & $3.90 \mathrm{e} 2$ & $2.61 \mathrm{e}-7$ & 0.97 & $5.68 \mathrm{e} 7$ & $3.03 \mathrm{e}-11$ \\
1000 & $1.31 \mathrm{e} 3$ & $1.89 \mathrm{e}-6$ & 1 & $5.23 \mathrm{e} 2$ & $7.76 \mathrm{e}-7$ & 0.96 & $4.16 \mathrm{e} 7$ & $3.76 \mathrm{e}-11$ \\
\hline
\end{tabular}


Table S5. Extracted equivalent circuit component values for bare ITO electrodes.

\begin{tabular}{ccccccccc}
\hline $\begin{array}{c}D \\
{[\mu \mathrm{m}]}\end{array}$ & $\begin{array}{c}R_{S}+R_{R} \\
{[\Omega]}\end{array}$ & $\begin{array}{c}Q_{d l} \\
{\left[\mathrm{~F} \cdot \mathrm{s}^{\mathrm{n}-1}\right]}\end{array}$ & $n_{d l}$ & $\begin{array}{c}R_{C} \\
{[\Omega]}\end{array}$ & $\begin{array}{c}Q_{a d} \\
{\left[\mathrm{~F} \cdot \mathrm{s}^{\mathrm{n}-1}\right]}\end{array}$ & $n_{a d}$ & $\begin{array}{c}R_{D} \\
{[\Omega]}\end{array}$ & $\begin{array}{c}C_{P} \\
{[\mathrm{~F}]}\end{array}$ \\
\hline 2.5 & $1.36 \mathrm{e} 5$ & $2.59 \mathrm{e}-11$ & 0.74 & $2.84 \mathrm{e} 8$ & $1.82 \mathrm{e}-11$ & 0.85 & $1.06 \mathrm{e} 13$ & $2.10 \mathrm{e}-12$ \\
5 & $6.75 \mathrm{e} 4$ & $4.47 \mathrm{e}-11$ & 0.78 & $7.55 \mathrm{e} 7$ & $2.63 \mathrm{e}-11$ & 0.85 & $1.01 \mathrm{e} 13$ & $1.56 \mathrm{e}-12$ \\
10 & $3.43 \mathrm{e} 4$ & $5.26 \mathrm{e}-12$ & 1 & $1.13 \mathrm{e} 6$ & $2.93 \mathrm{e}-11$ & 0.77 & $4.02 \mathrm{e} 10$ & $2.19 \mathrm{e}-12$ \\
15 & $2.48 \mathrm{e} 4$ & $1.03 \mathrm{e}-11$ & 1 & $1.27 \mathrm{e} 6$ & $4.27 \mathrm{e}-11$ & 0.75 & $5.71 \mathrm{e} 10$ & $2.08 \mathrm{e}-12$ \\
20 & $1.78 \mathrm{e} 4$ & $2.89 \mathrm{e}-11$ & 0.95 & $2.31 \mathrm{e} 6$ & $3.88 \mathrm{e}-11$ & 0.61 & $2.73 \mathrm{e} 12$ & $3.46 \mathrm{e}-12$ \\
50 & $8.51 \mathrm{e} 3$ & $1.76 \mathrm{e}-10$ & 0.94 & $6.05 \mathrm{e} 7$ & $1.78 \mathrm{e}-10$ & 0.67 & $6.10 \mathrm{e} 11$ & $8.09 \mathrm{e}-12$ \\
100 & $4.52 \mathrm{e} 3$ & $5.68 \mathrm{e}-10$ & 0.95 & $9.87 \mathrm{e} 6$ & $6.38 \mathrm{e}-10$ & 0.65 & $5.95 \mathrm{e} 11$ & $1.03 \mathrm{e}-11$ \\
200 & $2.86 \mathrm{e} 3$ & $1.93 \mathrm{e}-9$ & 0.95 & $1.33 \mathrm{e} 6$ & $2.07 \mathrm{e}-9$ & 0.59 & $4.95 \mathrm{e} 11$ & $3.29 \mathrm{e}-11$ \\
500 & $1.84 \mathrm{e} 3$ & $1.17 \mathrm{e}-8$ & 0.96 & $7.13 \mathrm{e} 5$ & $5.57 \mathrm{e}-9$ & 0.62 & $1.17 \mathrm{e} 9$ & $3.48 \mathrm{e}-11$ \\
1000 & $1.49 \mathrm{e} 3$ & $4.30 \mathrm{e}-8$ & 0.96 & $1.05 \mathrm{e} 4$ & $2.14 \mathrm{e}-8$ & 0.63 & $1.01 \mathrm{e} 9$ & $4.58 \mathrm{e}-11$ \\
\hline
\end{tabular}

Table S6. Extracted equivalent circuit component values for PEDOT:PSS/ITO electrodes.

\begin{tabular}{ccccccccc}
$\begin{array}{c}D \\
{[\mu \mathrm{m}]}\end{array}$ & $\begin{array}{c}R_{S}+R_{R} \\
{[\Omega]}\end{array}$ & $\begin{array}{c}Q_{d l} \\
{\left[\mathrm{~F} \cdot \mathrm{s}^{\mathrm{n}-1}\right]}\end{array}$ & $n_{d l}$ & $\begin{array}{c}R_{C} \\
{[\Omega]}\end{array}$ & $\begin{array}{c}Q_{a d} \\
{\left[\mathrm{~F} \cdot \mathrm{s}^{\mathrm{n}-1}\right]}\end{array}$ & $n_{a d}$ & $\begin{array}{c}R_{D} \\
{[\Omega]}\end{array}$ & $\begin{array}{c}C_{P} \\
{[\mathrm{~F}]}\end{array}$ \\
\hline 2.5 & $9.45 \mathrm{e} 4$ & $3.75 \mathrm{e}-11$ & 0.77 & $7.18 \mathrm{e} 8$ & $4.40 \mathrm{e}-11$ & 1 & $2.59 \mathrm{e} 10$ & $2.12 \mathrm{e}-12$ \\
5 & $4.78 \mathrm{e} 4$ & $2.71 \mathrm{e}-11$ & 0.82 & $8.93 \mathrm{e} 8$ & $4.62 \mathrm{e}-12$ & 1 & $2.39 \mathrm{e} 10$ & $2.47 \mathrm{e}-12$ \\
10 & $2.44 \mathrm{e} 4$ & $6.57 \mathrm{e}-11$ & 0.88 & $5.09 \mathrm{e} 7$ & $7.08 \mathrm{e}-10$ & 0.98 & $2.18 \mathrm{e} 10$ & $2.71 \mathrm{e}-12$ \\
15 & $1.58 \mathrm{e} 4$ & $2.20 \mathrm{e}-10$ & 0.85 & $1.84 \mathrm{e} 7$ & $1.53 \mathrm{e}-9$ & 0.95 & $2.10 \mathrm{e} 10$ & $2.72 \mathrm{e}-12$ \\
20 & $1.33 \mathrm{e} 4$ & $3.65 \mathrm{e}-10$ & 0.85 & $1.85 \mathrm{e} 7$ & $2.42 \mathrm{e}-9$ & 0.95 & $1.78 \mathrm{e} 10$ & $3.46 \mathrm{e}-12$ \\
50 & $8.04 \mathrm{e} 3$ & $1.74 \mathrm{e}-9$ & 0.88 & $2.44 \mathrm{e} 5$ & $1.20 \mathrm{e}-8$ & 1 & $1.48 \mathrm{e} 10$ & $4.81 \mathrm{e}-12$ \\
100 & $4.68 \mathrm{e} 3$ & $5.18 \mathrm{e}-9$ & 0.90 & $4.74 \mathrm{e} 4$ & $3.90 \mathrm{e}-8$ & 0.99 & $1.17 \mathrm{e} 10$ & $1.72 \mathrm{e}-11$ \\
200 & $2.82 \mathrm{e} 3$ & $2.31 \mathrm{e}-8$ & 0.89 & $6.17 \mathrm{e} 3$ & $1.11 \mathrm{e}-7$ & 1 & $1.00 \mathrm{e} 8$ & $2.14 \mathrm{e}-11$ \\
500 & $1.79 \mathrm{e} 3$ & $1.16 \mathrm{e}-7$ & 0.92 & $5.71 \mathrm{e} 2$ & $5.06 \mathrm{e}-7$ & 1 & $5.84 \mathrm{e} 7$ & $3.92 \mathrm{e}-11$ \\
1000 & $1.44 \mathrm{e} 3$ & $3.58 \mathrm{e}-7$ & 0.95 & $1.31 \mathrm{e} 2$ & $1.95 \mathrm{e}-6$ & 0.99 & $2.94 \mathrm{e} 7$ & $4.26 \mathrm{e}-11$ \\
\hline
\end{tabular}


Table S7. Extracted equivalent circuit component values for bare TiN electrodes.

\begin{tabular}{ccccccccc}
\hline $\begin{array}{c}D \\
{[\mu \mathrm{m}]}\end{array}$ & $\begin{array}{c}R_{S}+R_{R} \\
{[\Omega]}\end{array}$ & $\begin{array}{c}Q_{d l} \\
{\left[\mathrm{~F} \cdot \mathrm{s}^{\mathrm{n}-1}\right]}\end{array}$ & $n_{d l}$ & $\begin{array}{c}R_{C} \\
{[\Omega]}\end{array}$ & $\begin{array}{c}Q_{a d} \\
{\left[\mathrm{~F} \cdot \mathrm{s}^{\mathrm{n}-1}\right]}\end{array}$ & $n_{a d}$ & $\begin{array}{c}R_{D} \\
{[\Omega]}\end{array}$ & $\begin{array}{c}C_{P} \\
{[\mathrm{~F}]}\end{array}$ \\
\hline 2.5 & $1.49 \mathrm{e} 5$ & $1.85 \mathrm{e}-10$ & 0.60 & $1.71 \mathrm{e} 7$ & $2.97 \mathrm{e}-10$ & 0.80 & $1.24 \mathrm{e} 10$ & $2.66 \mathrm{e}-12$ \\
5 & $7.96 \mathrm{e} 4$ & $3.00 \mathrm{e}-10$ & 0.54 & $5.92 \mathrm{e} 6$ & $2.32 \mathrm{e}-10$ & 0.80 & $1.05 \mathrm{e} 10$ & $2.74 \mathrm{e}-12$ \\
10 & $4.33 \mathrm{e} 4$ & $9.36 \mathrm{e}-10$ & 0.67 & $2.75 \mathrm{e} 6$ & $2.58 \mathrm{e}-10$ & 0.89 & $5.52 \mathrm{e} 9$ & $4.97 \mathrm{e}-12$ \\
15 & $3.06 \mathrm{e} 4$ & $1.28 \mathrm{e}-9$ & 0.72 & $1.25 \mathrm{e} 6$ & $7.29 \mathrm{e}-10$ & 0.84 & $1.74 \mathrm{e} 9$ & $7.18 \mathrm{e}-12$ \\
20 & $2.50 \mathrm{e} 4$ & $4.10 \mathrm{e}-9$ & 0.79 & $2.21 \mathrm{e} 5$ & $2.88 \mathrm{e}-9$ & 0.83 & $2.10 \mathrm{e} 8$ & $9.81 \mathrm{e}-12$ \\
50 & $9.47 \mathrm{e} 3$ & $6.83 \mathrm{e}-9$ & 0.83 & $1.24 \mathrm{e} 5$ & $5.25 \mathrm{e}-9$ & 0.85 & $3.44 \mathrm{e} 8$ & $2.12 \mathrm{e}-11$ \\
100 & $4.97 \mathrm{e} 3$ & $1.89 \mathrm{e}-8$ & 0.87 & $2.23 \mathrm{e} 4$ & $1.85 \mathrm{e}-8$ & 0.87 & $1.29 \mathrm{e} 8$ & $3.59 \mathrm{e}-11$ \\
200 & $2.89 \mathrm{e} 3$ & $7.11 \mathrm{e}-8$ & 0.87 & $8.75 \mathrm{e} 3$ & $7.05 \mathrm{e}-8$ & 0.87 & $4.52 \mathrm{e} 7$ & $4.34 \mathrm{e}-11$ \\
500 & $1.73 \mathrm{e} 3$ & $3.82 \mathrm{e}-7$ & 0.89 & $1.78 \mathrm{e} 3$ & $4.53 \mathrm{e}-7$ & 0.87 & $7.35 \mathrm{e} 6$ & $4.94 \mathrm{e}-11$ \\
1000 & $1.38 \mathrm{e} 3$ & $1.40 \mathrm{e}-6$ & 0.89 & $6.02 \mathrm{e} 2$ & $1.85 \mathrm{e}-6$ & 0.88 & $2.03 \mathrm{e} 6$ & $4.93 \mathrm{e}-11$ \\
\hline
\end{tabular}

Table S8. Extracted equivalent circuit component values for PEDOT:PSS/TiN electrodes.

\begin{tabular}{|c|c|c|c|c|c|c|c|c|}
\hline $\begin{array}{c}D \\
{[\mu \mathrm{m}]}\end{array}$ & $\begin{array}{c}R_{S}+R_{R} \\
{[\Omega]}\end{array}$ & $\underset{\left[\mathrm{F} \cdot \mathrm{s}^{\mathrm{n}-1}\right]}{\left.Q_{d l}\right]}$ & $n_{d l}$ & $\begin{array}{c}R_{C} \\
{[\Omega]}\end{array}$ & $\underset{\left[\mathrm{F} \cdot \mathrm{s}^{\mathrm{n}-1}\right]}{Q_{a d}}$ & $n_{a d}$ & $\begin{array}{c}R_{D} \\
{[\Omega]}\end{array}$ & $\begin{array}{l}C_{P} \\
{[\mathrm{~F}]}\end{array}$ \\
\hline 2.5 & $1.93 \mathrm{e} 5$ & $1.72 \mathrm{e}-10$ & 0.90 & $9.76 \mathrm{e} 5$ & $1.65 \mathrm{e}-10$ & 0.98 & $2.53 \mathrm{e} 10$ & $5.56 \mathrm{e}-12$ \\
\hline 5 & $9.27 \mathrm{e} 4$ & $7.35 \mathrm{e}-10$ & 0.85 & $2.60 \mathrm{e} 8$ & $2.55 \mathrm{e}-10$ & 0.98 & $1.53 \mathrm{e} 10$ & $8.44 \mathrm{e}-12$ \\
\hline 10 & $4.02 \mathrm{e} 4$ & $1.97 \mathrm{e}-9$ & 0.87 & $4.02 \mathrm{e} 5$ & $1.44 \mathrm{e}-9$ & 0.91 & $2.00 \mathrm{e} 9$ & $1.25 \mathrm{e}-11$ \\
\hline 15 & $2.86 \mathrm{e} 4$ & $2.46 \mathrm{e}-9$ & 0.93 & $5.77 \mathrm{e} 4$ & $3.39 \mathrm{e}-9$ & 0.91 & $5.72 \mathrm{e} 8$ & $1.48 \mathrm{e}-11$ \\
\hline 20 & $1.99 \mathrm{e} 4$ & $2.97 \mathrm{e}-9$ & 0.95 & $1.80 \mathrm{e} 4$ & $6.60 \mathrm{e}-9$ & 0.90 & $1.84 \mathrm{e} 8$ & $1.35 \mathrm{e}-11$ \\
\hline 50 & $7.08 \mathrm{e} 3$ & $4.46 \mathrm{e}-8$ & 0.79 & $2.38 \mathrm{e} 3$ & $1.80 \mathrm{e}-8$ & 1 & $2.62 \mathrm{e} 8$ & $4.77 \mathrm{e}-12$ \\
\hline 100 & $4.11 \mathrm{e} 3$ & $1.91 \mathrm{e}-7$ & 0.87 & $2.30 \mathrm{e} 3$ & $2.63 \mathrm{e}-8$ & 1 & $1.60 \mathrm{e} 8$ & $2.23 \mathrm{e}-11$ \\
\hline 200 & $2.48 \mathrm{e} 3$ & $7.46 \mathrm{e}-7$ & 0.88 & $1.30 \mathrm{e} 3$ & $7.49 \mathrm{e}-8$ & 1 & $1.00 \mathrm{e} 8$ & $2.26 \mathrm{e}-11$ \\
\hline 500 & $1.55 \mathrm{e} 3$ & $4.08 \mathrm{e}-6$ & 0.92 & $5.58 \mathrm{e} 4$ & $5.69 \mathrm{e}-7$ & 1 & $4.62 \mathrm{e} 9$ & $3.39 \mathrm{e}-11$ \\
\hline 1000 & $1.23 \mathrm{e} 3$ & $1.58 \mathrm{e}-5$ & 0.92 & $7.50 \mathrm{e} 3$ & $1.94 \mathrm{e}-6$ & 1 & $4.19 \mathrm{e} 9$ & $4.07 \mathrm{e}-11$ \\
\hline
\end{tabular}



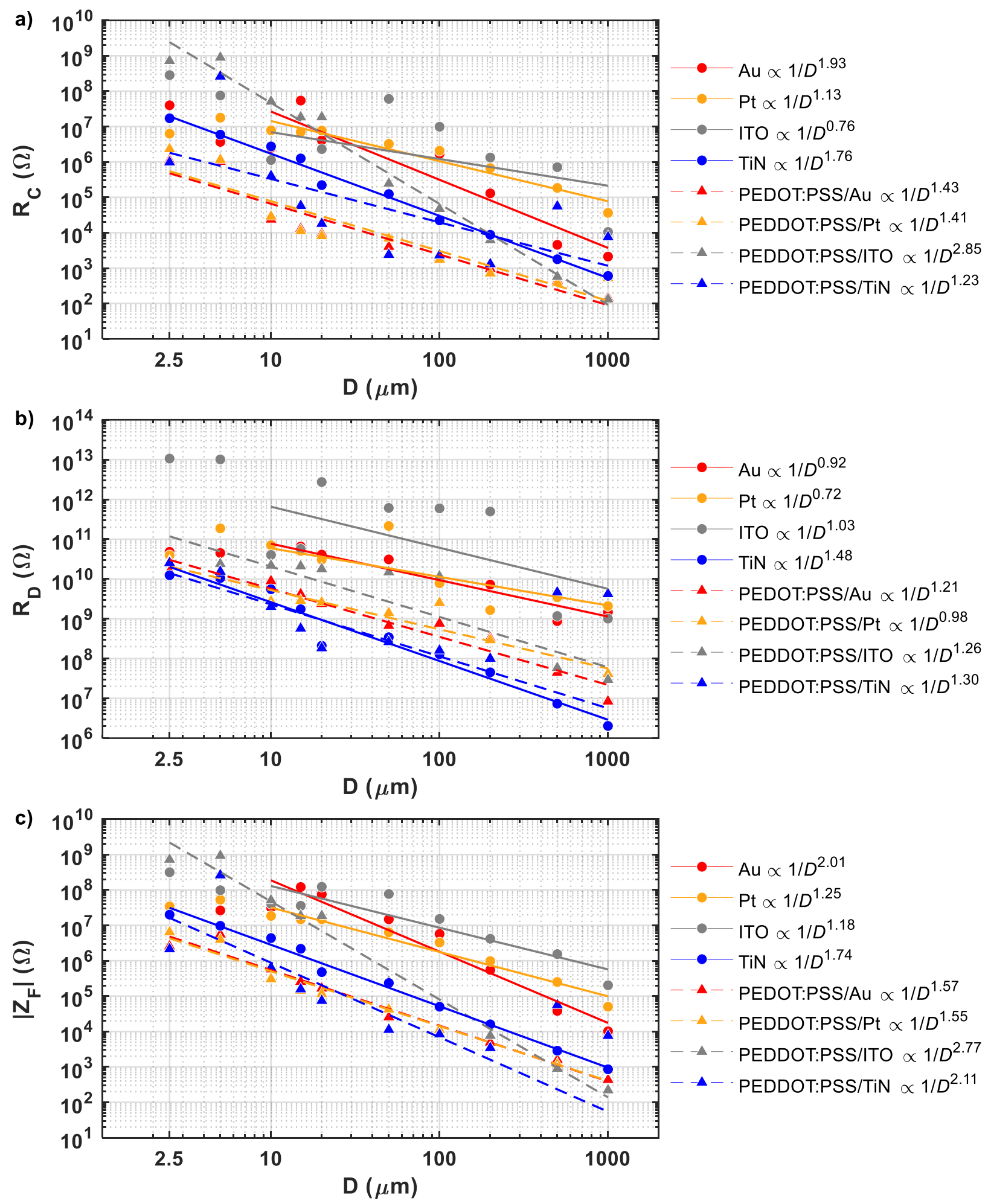

Figure S9. Scaling effects of electrode diameter on additional faradaic electrochemical elements. Size dependency of a) charge transfer resistance b) diffusion resistance, and c) combined faradaic impedance consisting of $R_{C}+\left(R_{D} \| Z_{a d}\right)$. 
a)

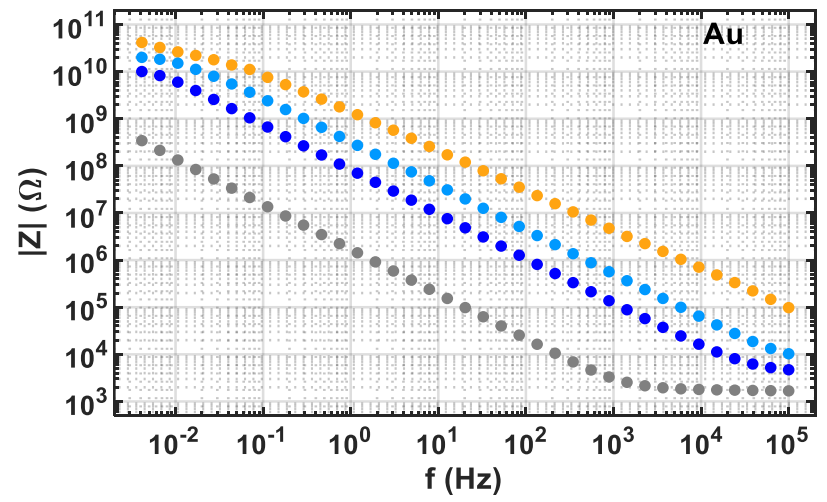

c)

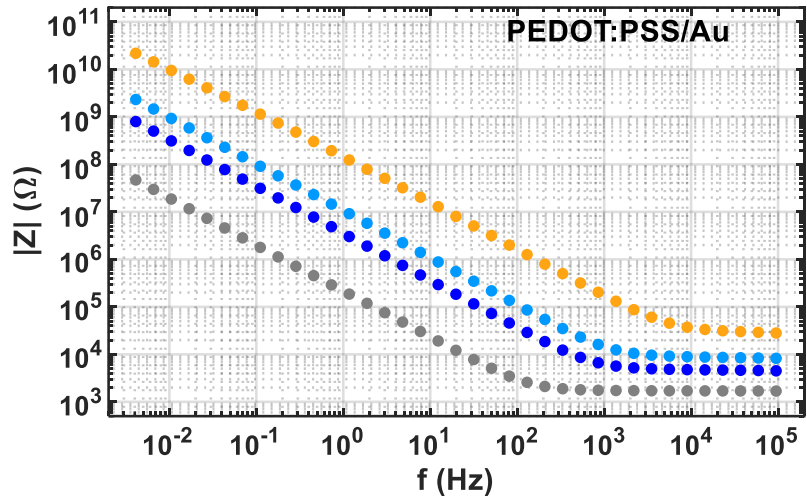

e)

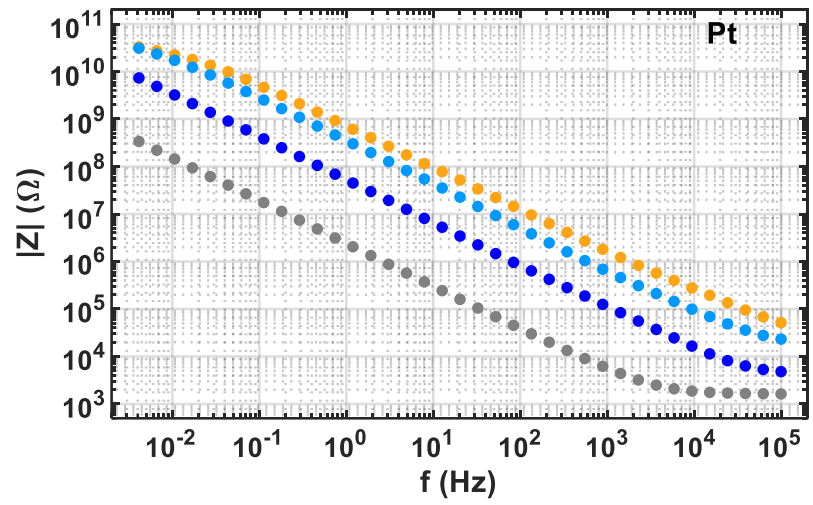

g)

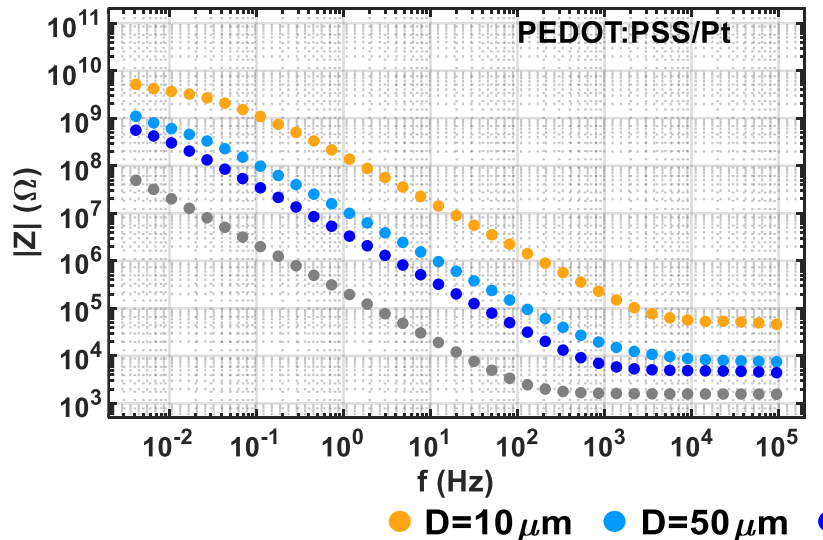

b)

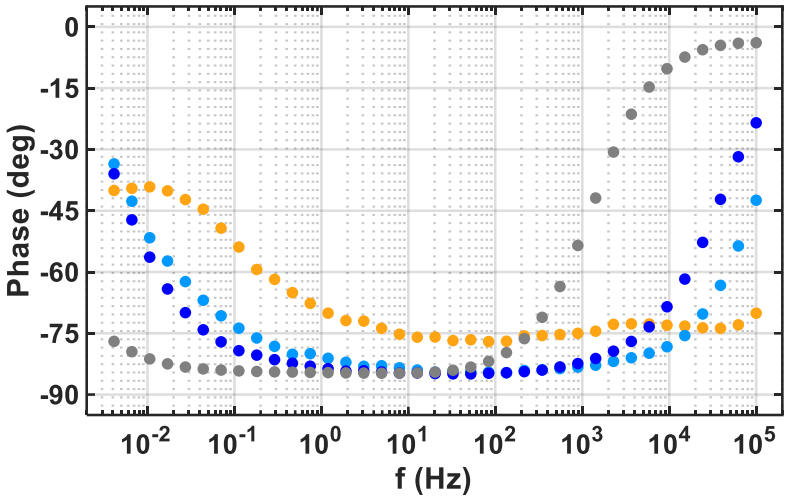

d)

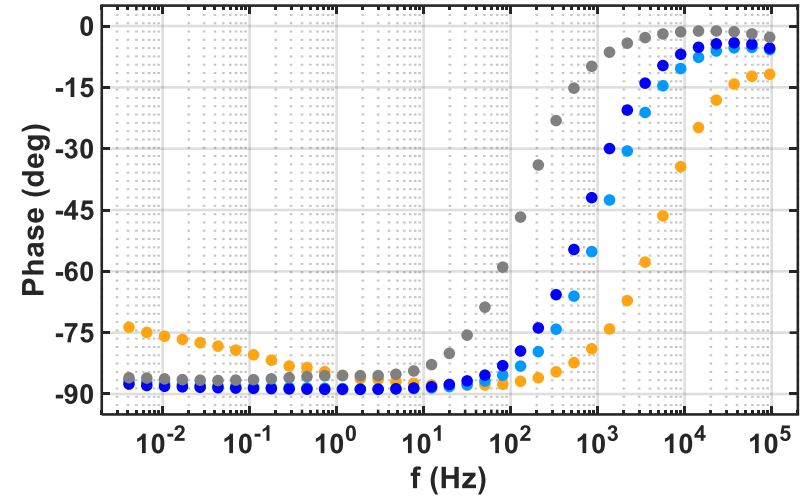

f)

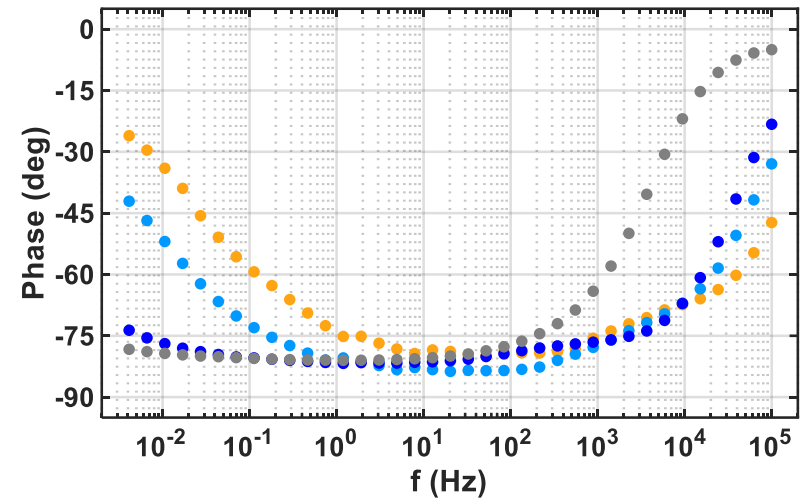

h)

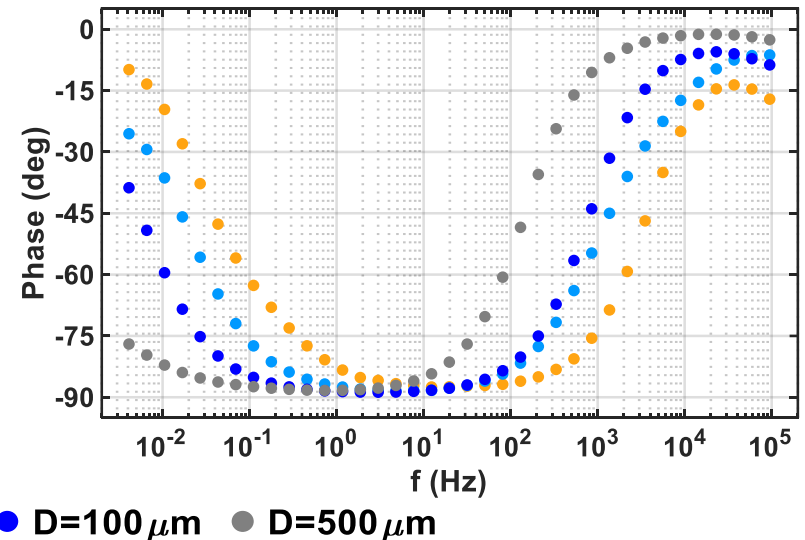


i)

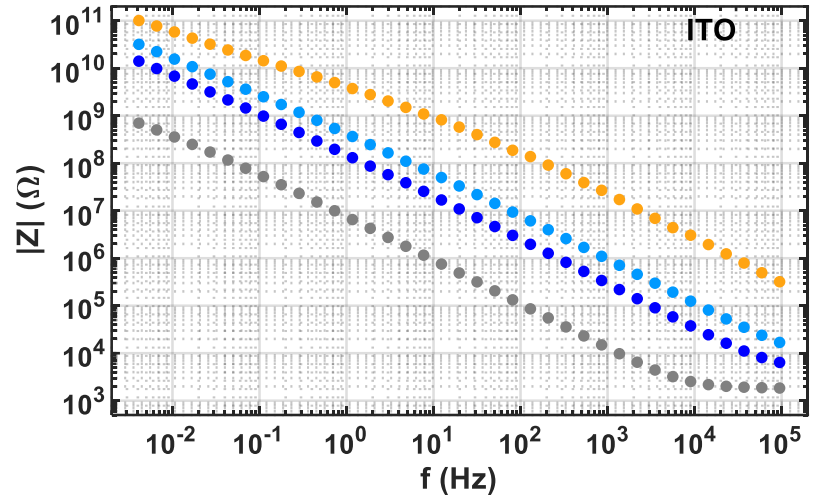

k)

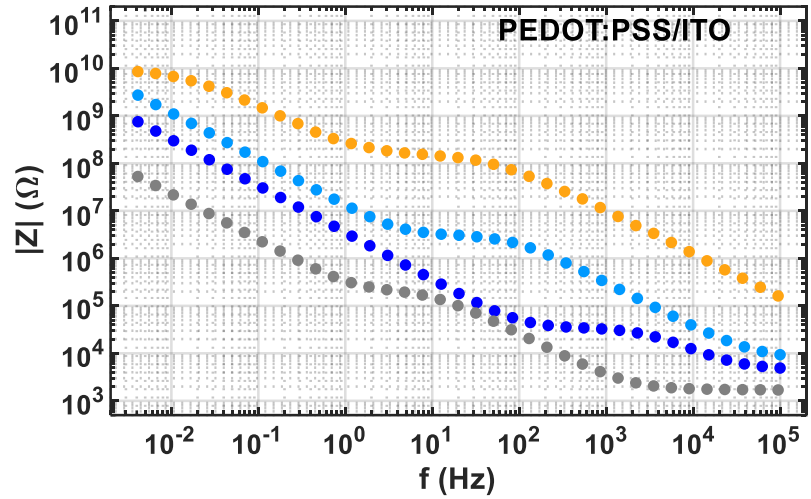

m)

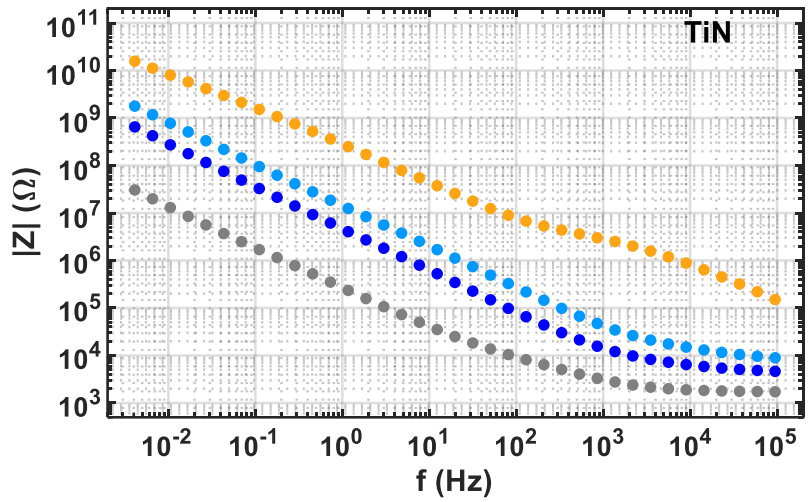

o)

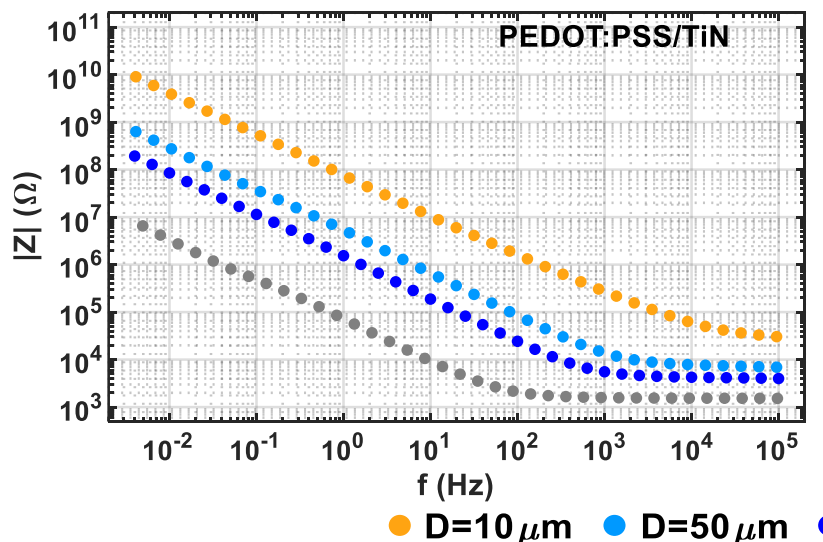

j)

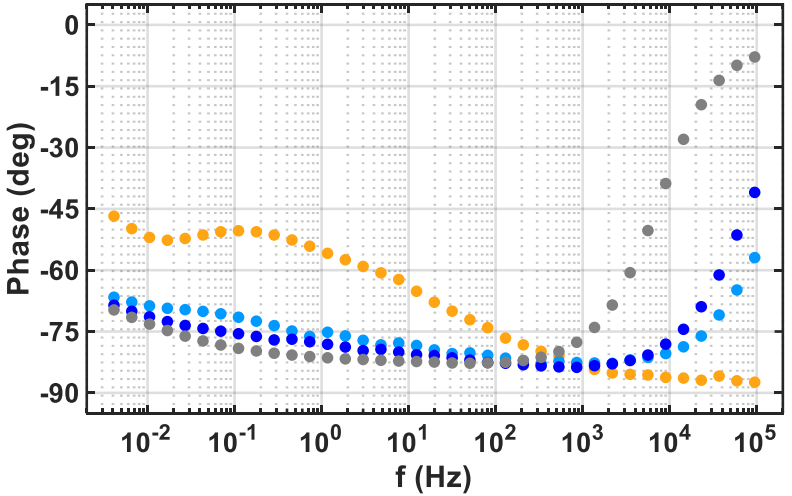

l)

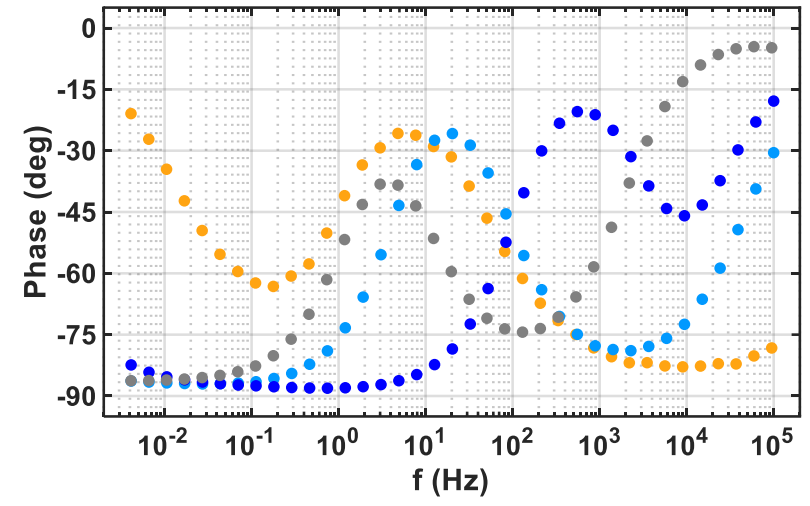

n)

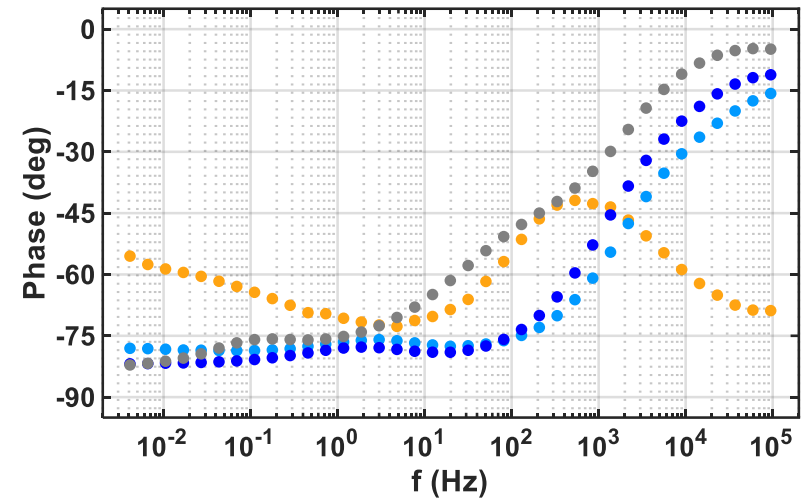

p)

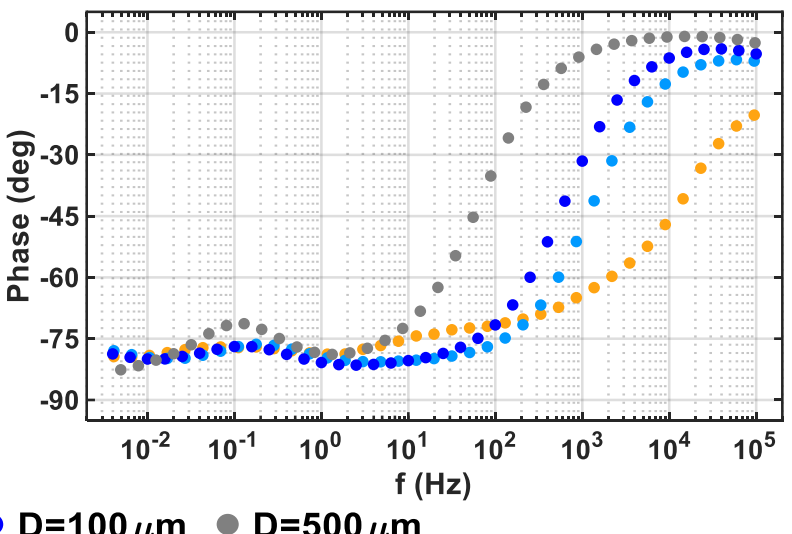


Figure S10. EIS plots of Au, Pt, TiN, and ITO electrodes with different sizes ranging from $D=10,50,100,500 \mu \mathrm{m}$ coated with and without PEDOT:PSS from $4 \mathrm{mHz}$ to $100 \mathrm{kHz}$. Impedance magnitude and phase plots, respectively, of a,b) $\mathrm{Au}, \mathbf{c}, \mathbf{d})$ PEDOT:PSS/Au, e,f) Pt, g.h) PEDOT:PSS/Pt electrodes, i,j) ITO, k,l) PEDOT:PSS/ITO, m,n) TiN, and o,p) PEDOT:PSS/TiN electrodes. 
a)

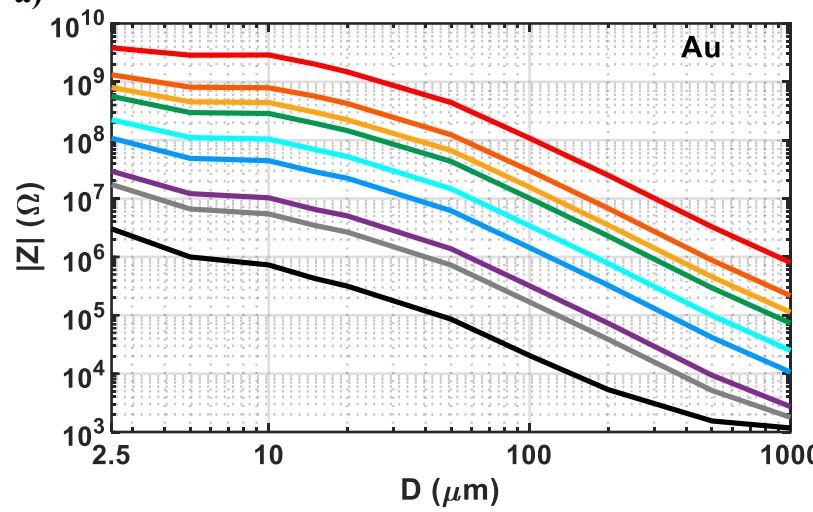

c)

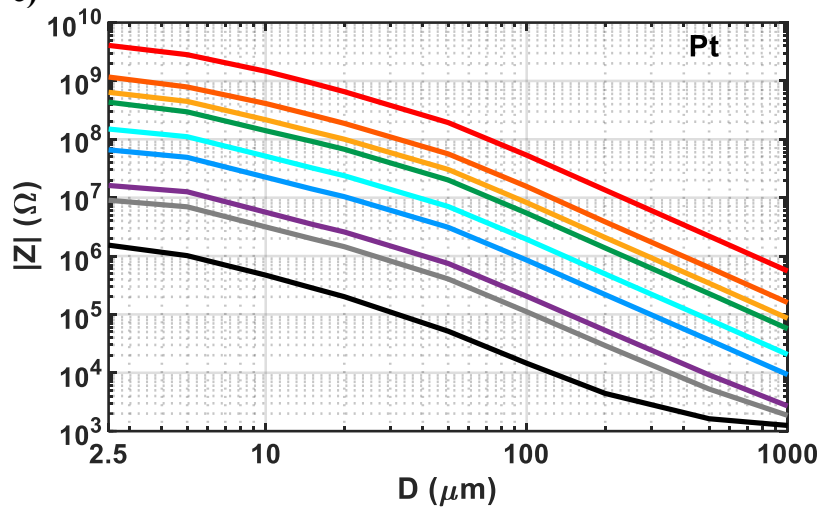

e)

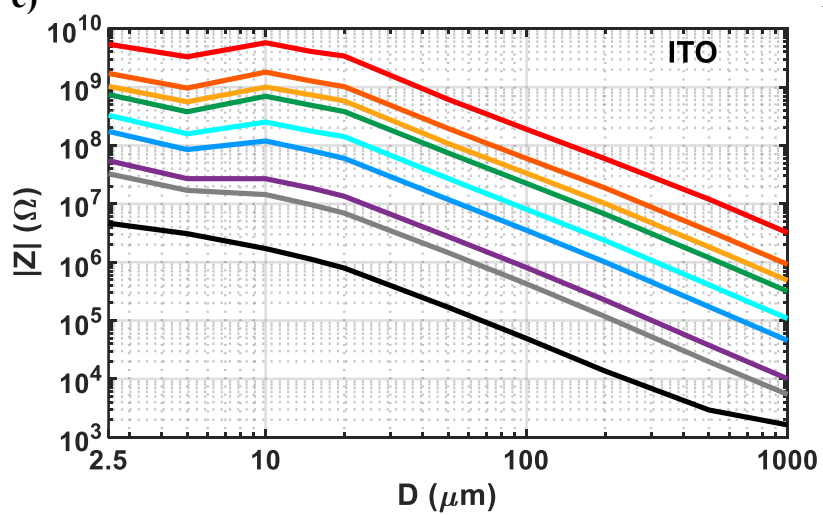

g)

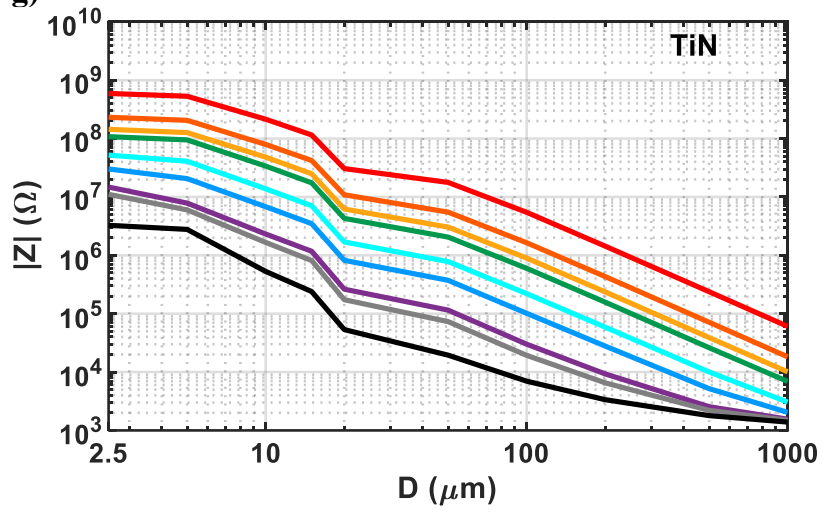

b)

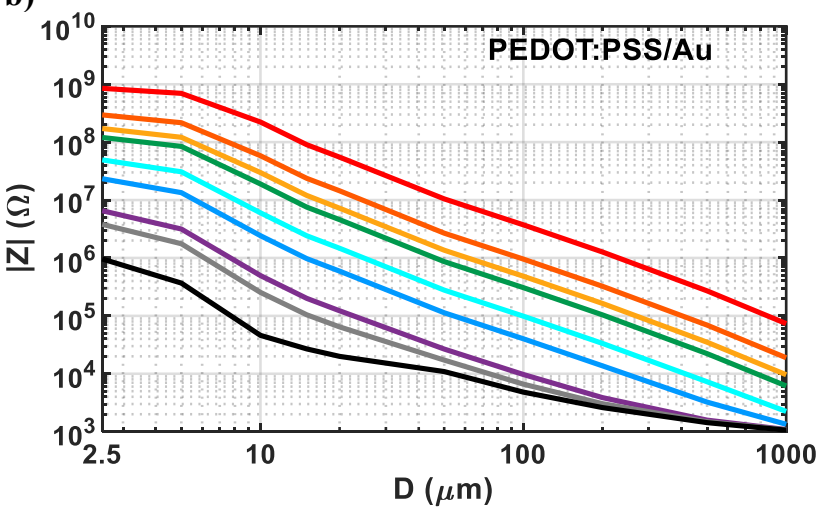

d)

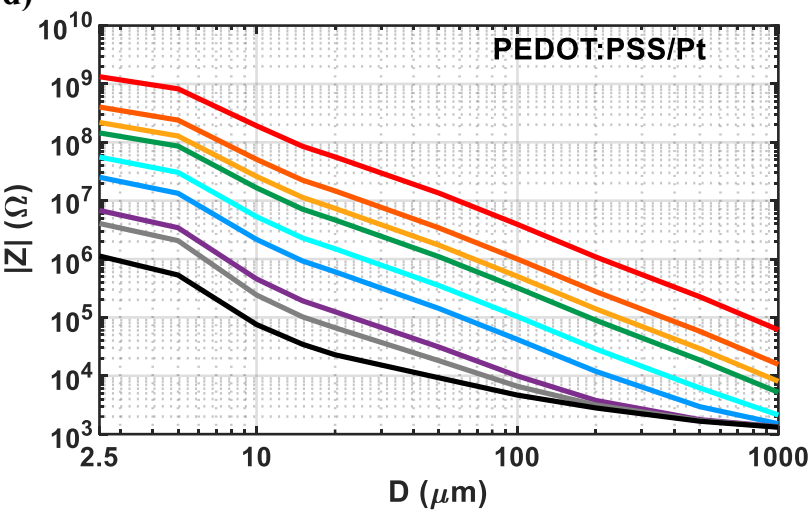

f)

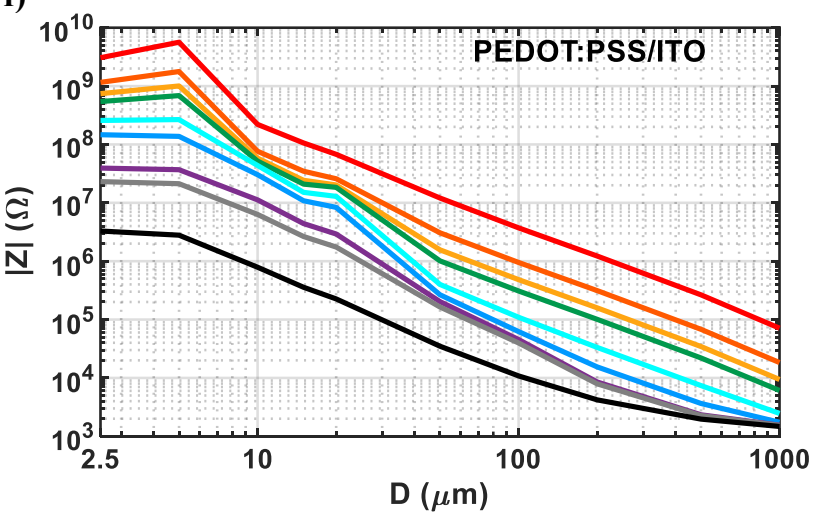

h)

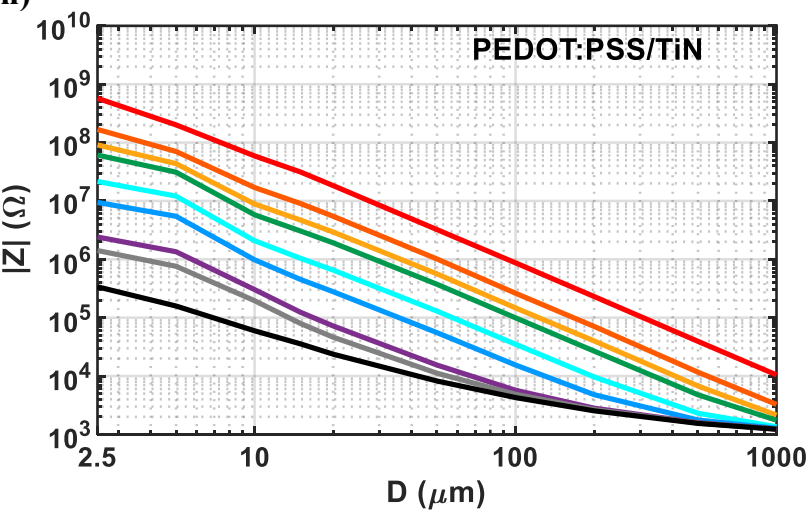

$-1 \mathrm{~Hz}-4 \mathrm{~Hz}-8 \mathrm{~Hz}-12 \mathrm{~Hz}-40 \mathrm{~Hz}$ $100 \mathrm{~Hz}-500 \mathrm{~Hz}-1 \mathrm{kHz}$ $10 \mathrm{kHz}$ 
Figure S11. EIS plotted at different biologically relevant frequencies of Au, Pt. ITO, and TiN electrodes coated with and without PEDOT:PSS. Impedance magnitude plots, respectively, of a) $\mathrm{Au}$, b) PEDOT:PSS/Au, c) Pt, d) PEDOT:PSS/Pt electrodes, e) ITO, f) PEDOT:PSS/ITO, g) TiN, and h) PEDOT:PSS/TiN electrodes. 
a) NP7-3000P Spincoating

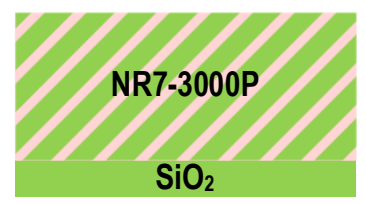

b) Photolithography, Metal Deposition

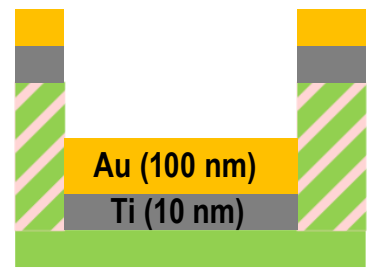

c) Photolithography, Electrode Deposition

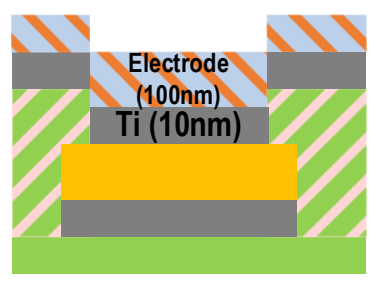

d) Parylene Passivation

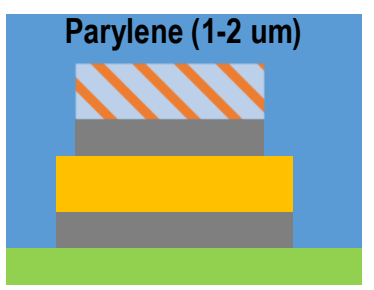

e) Photolithography, Etch Opening

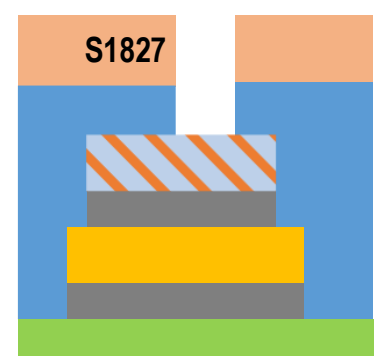

\section{f) Spincoat Pedot:PSS}

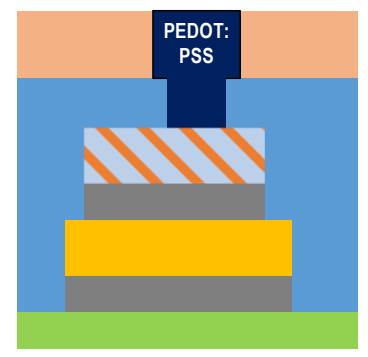

g) Liftoff

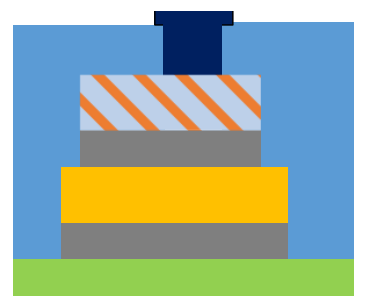

h) Vessel Attachment to Wafer

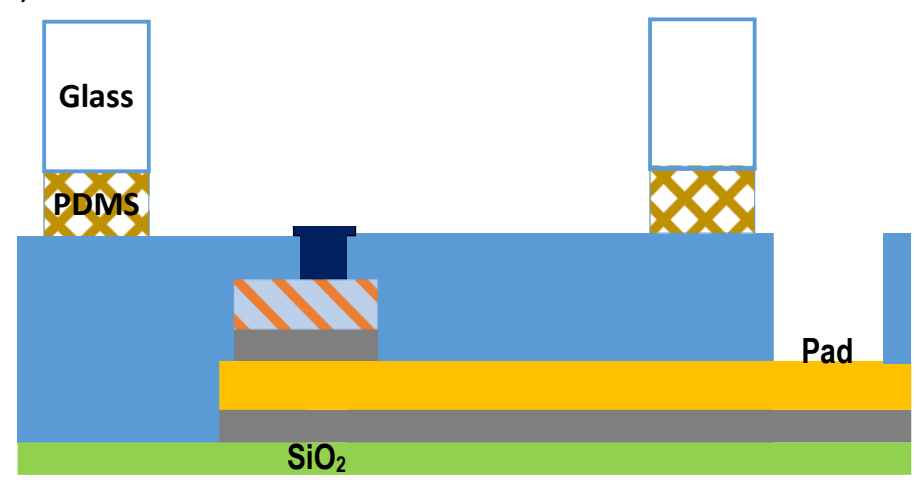

i) Output Pin Attachment

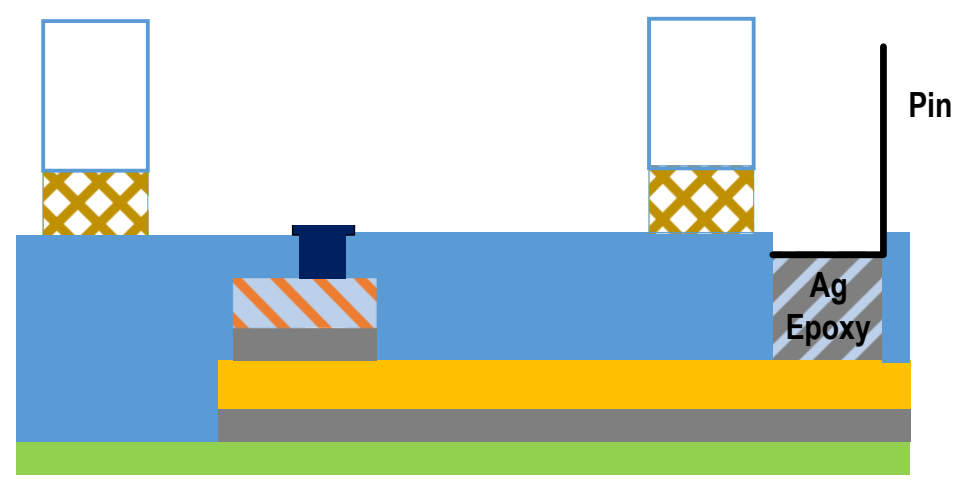

Figure S12. Device fabrication process. a) Spin coating of photoresist, b) gold seed and routing sputtering with photoresist mask followed by liftoff, c) electrode material sputtering with photoresist mask followed by liftoff, d) parylene passivation, e) electrode opening, d) spin coating of PEDOT:PSS, g) liftoff of PEDOT:PSS, h) attachment of electrolyte vessel, i) attachment of output pin. 


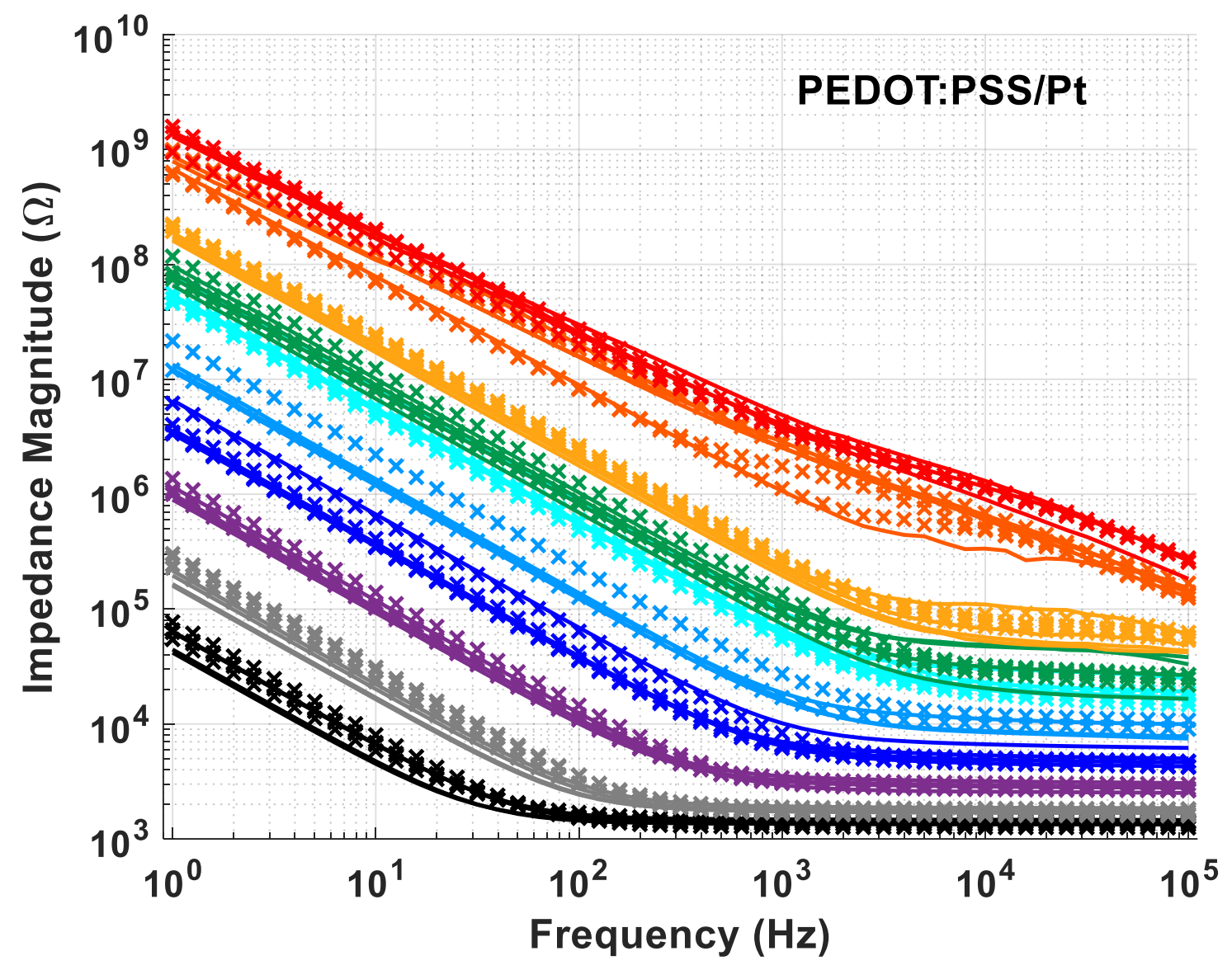

$\bullet \mathrm{D}=2.5 \mu \mathrm{m} \bullet \mathrm{D}=5 \mu \mathrm{m} \bullet \mathrm{D}=10 \mu \mathrm{m} \bullet \mathrm{D}=15 \mu \mathrm{m} \bullet \mathrm{D}=20 \mu \mathrm{m} \bullet \mathrm{D}=50 \mu \mathrm{m} \bullet \mathrm{D}=100 \mu \mathrm{m} \bullet \mathrm{D}=200 \mu \mathrm{m} \bullet \mathrm{D}=500 \mu \mathrm{m} \bullet \mathrm{D}=1000 \mu \mathrm{m}$ Figure S13. Spread of data for PEDOT:PSS/Pt electrodes. The impedance of the electrodes between device-to-device is similar. Solid lines represent one set of electrodes from a device while " $\times$ " represent a set from the second device. 